\title{
A Theoretical Study of Piezoelectricity, Phase Stability, and Surface Diffusion in Disordered Multicomponent Nitrides
}

\author{
Christopher Tholander
}

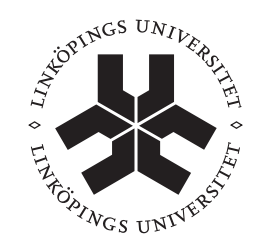

\section{Linköpings universitet} INSTITUTE OF TECHNOLOGY

Thin Film Physics Division Department of Physics, Chemistry, and Biology (IFM) Linköping University, SE-581 83 Linköping, Sweden 
(C) Christopher Tholander

ISBN 978-91-7519-253-6

ISSN 0280-7971

Printed by LiU-Tryck 2014 
Disordered multicomponent nitride thin film can be used for various applications. The focus of this Licentiate Thesis lies on the theoretical study of piezoelectric properties, phase stability and surface diffusion in multifunctional hard coating nitrides using density functional theory (DFT).

Piezoelectric thin films show great promise for microelectromechanical systems (MEMS), such as surface acoustic wave resonators or energy harvesters. One of the main benefits of nitride based piezoelectric devices is the much higher thermal stability compared to the commonly used lead zirconate titanate (PZT) based materials. This makes the nitride based material more suitable for application in, e.g., jet engines.

The discovery that alloying AlN with $\mathrm{ScN}$ can increase the piezoelectric response more than $500 \%$ due to a phase competition between the wurtzite phase in $\mathrm{AlN}$ and the hexagonal phase in $\mathrm{ScN}$, provides a fundamental basis for constructing highly responsive piezoelectric thin films. This approach was utilized on the neighboring nitride binaries, where $\mathrm{ScN}$ or $\mathrm{YN}$ was alloyed with $\mathrm{AlN}$, GaN, or InN. It established the general role of volume matching the binaries to easily achieve a structural instability in order to obtain a maximum increase of the piezoelectric response. For $\mathrm{Sc}_{0.5} \mathrm{Ga}_{0.5} \mathrm{~N}$ this increase is more than $900 \%$, compared to GaN. $\mathrm{Y}_{1-x} \operatorname{In}_{x} \mathrm{~N}$ is, however, the most promising alloy with the highest resulting piezoelectric response seconded only by $\mathrm{Sc}_{0.5} \mathrm{Al}_{0.5} \mathrm{~N}$.

Phase stability and lattice parameters (stress-strain states) of the $\mathrm{Y}_{1-x} \mathrm{Al}_{x} \mathrm{~N}$ alloy have been calculated in combination with experimental synthesis.

Hard protective coatings based on nitride thin films have been used in industrial applications for a long time. Two of the most successful coatings are TiN and the metastable $\mathrm{Ti}_{1-x} \mathrm{Al}_{x} \mathrm{~N}$. Although these two materials have been extensively investigated both experimentally and theoretically, at the atomic level little is known about $\mathrm{Ti}_{1-x} \mathrm{Al}_{x} \mathrm{~N}$ diffusion properties. This is in large part due to problems with configurational disorder in the alloy, because $\mathrm{Ti}$ and $\mathrm{Al}$ atoms are placed randomly at cation positions in the lattice, considerably increasing the complexity of the problem. To deal with this issues, we have used special quasi-random structure (SQS) models, as well as studying dilute concentrations of Al.

One of the most important mechanisms related to the growth of $\mathrm{Ti}_{1-x} \mathrm{Al}_{x} \mathrm{~N}$ is surface diffusion. Because $\mathrm{Ti}_{1-x} \mathrm{Al}_{x} \mathrm{~N}$ is a metastable material it has to be grown 
as a thin film with methods such as physical vapor deposition (PVD), in which surface diffusion plays a pivotal role in determining the microstructure evolution of the film.

In this work, the surface energetics and mobility of $\mathrm{Ti}$ and $\mathrm{Al}$ adatoms on a disordered $\mathrm{Ti}_{0.5} \mathrm{Al}_{0.5} \mathrm{~N}(001)$ surface are studied. Also the effects on the adatom energetics of $\mathrm{Ti}, \mathrm{Al}$, and $\mathrm{N}$ by the substitution of one Ti with an $\mathrm{Al}$ surface atom in $\operatorname{TiN}\left(\begin{array}{lll}0 & 0 & 1\end{array}\right), \operatorname{TiN}\left(\begin{array}{lll}0 & 1 & 1\end{array}\right)$, and $\operatorname{TiN}\left(\begin{array}{lll}1 & 1 & 1\end{array}\right)$ surfaces is studied. This provides an indepth atomistic understanding of how the energetics behind surface diffusion changes as TiN transitions into $\mathrm{Ti}_{0.5} \mathrm{Al}_{0.5} \mathrm{~N}$.

The investigations revealed many interesting results. i) That Ti adatom mobilities are dramatically reduced on the $\mathrm{TiN}$ and $\mathrm{Ti}_{0.5} \mathrm{Al}_{0.5} \mathrm{~N}(001)$ surfaces while $\mathrm{Al}$ adatoms are largely unaffected. ii) The reverse effect is found on the $\operatorname{TiN}(111)$ surface, $\mathrm{Al}$ adatom migration is reduced while Ti adatom migration is unaffected. iii) The magnetic spin polarization of $\mathrm{Ti}$ adatoms is shown to have an important effect on binding energies and diffusion path, e.g., the adsorption energy at bulk sites is increased by $0.14 \mathrm{eV}$. 
First of all, I would like to thank my main supervisor Dr. Björn Alling for the massive support during these first years of my Ph.D. studies. I've learned a tremendous amount from you already, and I'm looking forward to learning even more.

I would also like to thank my assistant supervisors Prof. Lars Hultman and Dr. Ferenc Tasnádi. Their contributions have been invaluable.

I am also grateful to Prof. Igor Abrikosov who introduced me to theoretical physics by giving me my first project when I was a Masters student.

A huge amount of thanks to all my coauthors who I have had the pleasure working with and learned so much from. Especially Dr. Agnė Žukauskaitè, our theoretical and experimental collaborations have proved to be exceptionally fruitful.

A special thanks goes to the lunch group, for providing friendly company and lunch conversations which have been entertaining and, at times, enlightening.

Thanks also to Jonas Saarimäki, who is always teaching me new painful ways to physically exhaust myself. It's been a pleasure.

One of my most important thanks goes to my family who have always been supportive. I would never have gotten this far without you.

Finally, I would like to thank my Malin for being so extraordinary wonderful. I'm immensely lucky to have you in my life. 

1 Introduction 1

1.1 Theoretical materials science . . . . . . . . . . . . . . . 2

1.2 Thin films . . . . . . . . . . . . . . . . . . . 3

1.3 Microscopic view of thin film growth . . . . . . . . . . . . . . . 3

1.4 Piezoelectric coatings . . . . . . . . . . . . . . . . . . 4

1.5 Hard coatings .................... 5

2 Methods $\quad 7$

2.1 Modeling atomic and electronic structure . . . . . . . . . . 7

2.2 Density functional theory . . . . . . . . . . . . . . . . . . . . . . . . . . .

2.2.1 Hohenberg-Kohn . . . . . . . . . . . . . . . . . . . . . 9

2.2 Kohn-Sham . . . . . . . . . . . . . . . . . . . 9

2.2.3 Local-density approximation . . . . . . . . . . . . . . . 10

2.2.4 Generalized gradient approximation . . . . . . . . . . 11

2.2.5 Beyond LDA and GGA . . . . . . . . . . . . . . 11

2.2 .6 Basis sets . . . . . . . . . . . . . . 12

2.3 Modeling random alloys . . . . . . . . . . . . . . . . . . . . . . 13

2.3.1 Special quasi-random structure model . . . . . . . . . . . . 14

2.3.2 Limitations of the special quasi-random structure model ................... . . 15

2.4 Phase stability . . . . . . . . . . . . . . . . . . 16

2.4.1 Approximate calculations for non-equilibrium conditions . . 17

2.5 Modeling elastic and piezoelectric properties . . . . . . . . . . . 18

2.5.1 Elastic properties . . . . . . . . . . . . . . 18

2.5.2 Piezoelectric properties . . . . . . . . . . . . 20

2.5.3 Berry-phase theory of polarization . . . . . . . . . . . 22

2.5.4 Piezoelectric response $d \ldots \ldots . \ldots . \ldots . \ldots 23$

2.6 Modeling diffusion . . . . . . . . . . . . . . . . . . 24 
2.6.1 Fick's first law . . . . . . . . . . . . . . . . . 24

2.6.2 Fick's second law . . . . . . . . . . . . . . . . 26

2.6.3 Fick's laws in more dimensions . . . . . . . . . . . . . . . 27

2.6.4 Temperature dependence . . . . . . . . . . . . 28

2.6.5 Calculating the diffusion activation barrier $\Delta E \ldots \ldots 28$

2.6.6 Approximating the diffusion prefactor . . . . . . . . . 30

2.6.7 Calculating the diffusivity on surfaces when there is more than one type of binding site . . . . . . . . . 31

3 Results and included pappers $\quad \mathbf{4 5}$

3.1 List of publications . . . . . . . . . . . . . . . . . . . . 46

3.2 Summary of included papers . . . . . . . . . . . . 47

$\begin{array}{ll}\text { Paper I } & 49\end{array}$

$\begin{array}{ll}\text { Paper II } & 51\end{array}$

$\begin{array}{lc}\text { Paper III } & 53\end{array}$

$\begin{array}{ll}\text { Paper IV } & 55\end{array}$ 


\section{CHAPTER 1}

\section{Introduction}

Materials have been a key part in the history of mankind, to such extent that historians and archeologists have seen it fit to divide our history into ages named after the prominent material used or introduced during that age.

The stone age began more than 2.5 million years ago. The first stone tools appeared as early as 3.36 million years ago [1], even before the advent of the Homo genus. It is divided into three phases, Paleolithic (Old stone age), Mesolithic (Middle stone age), and Neolithic (New stone age) [2]. Stone, e.g., flint, is a very hard material but it is also brittle, meaning it will break without significant deformation. Therefore, striking flint with an other stone causes flakes to detach from it rather than just creating dents, so that sharp cutting tools can be created. Metal use began during the neolithic phase, where copper, silver, and gold was used. Metals can be worked in to many forms because it can be soften by heating and hardened by hammering. Because these metals were rare and soft compared to stone, tools were still made of stone while metals were mainly used to create ornaments or decorations.

The first of the metal ages is the bronze age. The first phase of the bronze age is the Chalcolithic (Copper-Stone age). During this period the copper use becomes increasingly important, in part because copper was the most abundant of the accessible metals. However, the hardness of copper was not enough and ways to improve it was needed. This was done by alloying copper with other metals. Some were less successful than others. For example, alloying copper with arsenic does improve the material properties, however, the fumes from the process are deadly. Eventually, the combination copper and tin (bronze) was found to be the most successful, being harder and less brittles than pure copper. Of course even harder materials were sought for.

The next age was the iron age, which started in different parts of the world around 1500 to 1000 B.C [2]. A difficulty with iron is the high melting temperature. 
Therefore, it was initially obtained as a slag product, called bloom, from copper smelting operations. Iron in this form is porous, and workable at much lower temperatures. Moreover, pure iron is not harder than bronze so it had to be alloyed with an other material before there was an advantage to use it. The most successful alloys incorporated different amounts of carbon, creating different types of steel.

Worth to point out is that the search for new materials so far had been empirical, and many believed magic was part of the process. Not until the 19th and 20th century, was there a systematic approach to the search for new materials to a degree which could be called a material science. To a large part because we began to understand materials at an atomic level, discovering the fundamentals of chemistry and the proton, neutron, and electron. This was also when electronic materials emerged to a greater extent, becoming a pivotal part in 20th and 21st century materials science.

\subsection{Theoretical materials science}

Searching for new and improved materials is no simple task. If we allow there to be any number of components at any concentrations in our material, then there is an infinite amount of ways to combine the elements of the periodic table into different alloys. Making each of these materials with different synthesis routs and testing the properties of each one of them would certainly take an unbearable long time and require vast resources. Although there are ways to reduce the number of potential combinations using carefully designed experimental series, the amount of possibilities are still numerous. Therefore, it is important that we understand how materials work at an atomic and electronic level, and let that knowledge guide our search for better materials. The accumulation and systematization of this understanding is the theoretical part of materials science, which during the last couple of decades has been accelerated enormously using computer simulations.

Given that the theoretical framework is accurate enough, there are plenty of advantages with computer simulations, compared to experiments. It is possible to run simulations with toxic or radioactive materials, without posing a health risk. It is also possible to test expensive materials without any cost. Of course, there are also limitations to computer simulations. There is often a choice to be made wether a simulation should be accurate (e.g. include quantum effects) or if it should be large (e.g. include many atoms or long time scales). However, computers improve at an amazing rate, making them faster and able to handle more complex problems. Thus, we are continuously able to push the boundaries of what we can simulate even further. Continued development of the accuracy of the theoretical methods goes hand in hand with computations of specific materials research topics. This work is a piece in that big puzzle, by extending the frontiers of disordered multicomponent nitrides, discovering novel piezoelectric materials, and further the research on growth related features, i.e. surface diffusion and phase stability. 


\subsection{Thin films}

Not all advances in materials science have emerged from improving a material's properties by alloying or applying new elements. One advance of particular interest in this thesis comes from the fairly simple idea that the surface properties of a material can be improved by coating it with an other material, with good surface properties, but not necessarily good bulk properties. This allows us to combine the bulk properties of one material with the surface properties of another. For example, we can coat materials with a thin film to improve its oxidation resistance or wear resistance, or simply as a purely decorative coating. These coating does not even have to be very thick. What we characterize as a thin film ranges from a single monolayer in thickness to a few micrometers, which roughly corresponds to 25000 atomic layers. Although this is not thick enough for us to be able to see the cross section with our naked eye, it is enough to greatly improve the surface properties of a bulk material. Also, in this thin film form, due to the particularities of thin film synthesis methods, it is possible to grow materials which are thermodynamically only metastable, and in some cases impossible to create in bulk form.

The difficulty with metastable thin films is creating them. It is not just a matter of mixing liquid metal and poring it in a mold. Instead, other methods need to be utilized, e.g., physical vapor deposition (PVD), and chemical vapor deposition (CVD). The basic principle of these two methods is to deposit the thin film on top of a substrate at a temperature low enough for a desired metastable material to avoid transforming into its thermodynamically stable phases. This limits the kinetics of the adatoms, impinging on the surface of the growing film, so that they can not rearrange enough to reach the ground state structure.

A typical PVD process is high vacuum sputtering, where the components are ejected from a target source on to a substrate. Usually by using an inert gas such as argon to sputter free ions from the target. There are many different techniques of sputtering for improving the process and the quality of the films, e.g., using magnetrons, reactive gases or biasing the substrate.

In a typical CVD process, the chemistry is more important. The materials needed for the film are carried by volatile precursors, which react in such a way that they deposit the material on the surface. Although this method is typically suited for large scale, large area depositions, the films tend to be rougher compared to PVD films. However, the CVD process typically requires higher temperatures than PVD, which makes metastable thin film synthesis much more difficult [3].

\subsection{Microscopic view of thin film growth}

The surface diffusion, i.e. movement, of the adatoms and admolecules on a substrate, and subsequently layers of the film, is very important during both PVD and CVD growth because it governs the evolution of the film. If the rate at which atoms are added to the surface is low, then there is time for the adatoms already at the surface to arrange themselves and make each layer very flat and even. In the opposite case, when the rate of arriving atoms is very high, the films will become rough and possibly porous. This is because adatoms might start to form many 
small islands instead of few large ones, and there will not be enough time to fill in the voids between them before the next layer starts to grow.

There are many aspects that influences adatom surface diffusion other than the surface material and the adatom species, e.g., surface crystal orientation, and impurities. The adatom movement can differ a lot between surface orientations [4]. This can have an effect on the preferred orientation of the film, promoting one direction over another [5].

During alloy growth, there are multiple adatom species diffusing at the same time. If there is a large variation in, for example, how fast they diffuse on different surface orientations; then the composition could vary between grains ${ }^{1}$ resulting in inhomogeneous films. It is important to understand how different adatoms diffuse, in order to either promote or counter effects like this.

Currently much is known about the surface diffusion on single metal surfaces, and to a large extent also binaries. However, very little is known about many component alloys. Largely because of added complexity from configurational disorder. Also effects of impurities in multicomponent alloys are mostly unknown, although they can effect adatom diffusion in many different ways, promoting or obstructing their diffusion path. It is therefore important to study them, as is further discussed in Sec. 2.6 and in Papers III and IV.

\subsection{Piezoelectric coatings}

Piezoelectric materials have the property that they can convert vibrational to electrical energy, or the opposite, electrical energy into vibrations. This property is used in a wide range of microelectromechanical systems (MEMS), e.g., sensors, actuators, and energy harvesters to power micro-sized devices [6-10].
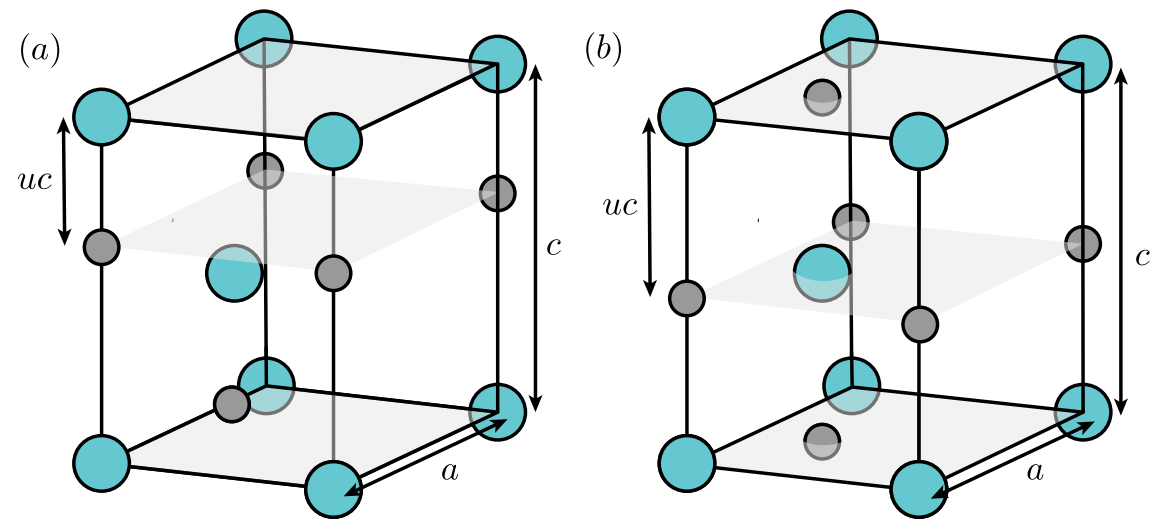

Figure 1.1. (a) The wurtzite crystal structure, with the in plane lattice parameter $a$, out of plane parameter $c$, and internal parameter $u$. (b) Layered hexagonal crystal structure. The difference between (a) and (b) is a that in (b) $u$ is always 0.5.

\footnotetext{
${ }^{1}$ Volumes with different crystal orientations.
} 
The reason why some materials exhibit a piezoelectric effect is because it changes its polarization upon deformation. Polarization comes from a shift between the positively and negatively charged ion position in the lattice (see Fig. 1.1). When mechanical strain is applied in a direction which increases or decreases the shift between the ions, there will be a flow of electrons to counteract the change in polarization. The reverse piezoelectric effect is simply the process in reverse, an applied voltage will force a shift of the ions to counteract the voltage.

Common piezoelectric devices are made of lead zirconium titanate (PZT), with the chemical formula $\mathrm{Pb}\left[\mathrm{Zr}_{x} \mathrm{Ti}_{1-x}\right] \mathrm{O}_{3}$. Although this material has a high piezoelectric response, $410 \mathrm{pC} / \mathrm{N}$ [11], it is limited by its maximum operational temperature of $250^{\circ} \mathrm{C}$ [12]. Generally there is a serious tradeoff between high piezoelectric response and high maximum use temperature [11]. For high temperature operations, in for example aircraft sensors, AlN can be used instead. AlN has a maximum operational temperature of $1150^{\circ} \mathrm{C}$ [13], 4.6 times higher than PZT.

$\mathrm{AlN}$ is a piezoelectric material in its stable wurtzite phase (see Fig.1.1). The shift between the $\mathrm{Al}$ and $\mathrm{N}$ ions create a polarization of the material. The $u$-value is used as a measure of the shift between the ions. Stress in the $c$-direction will change the $u$-value and cause piezoelectric effect.

By alloying AlN with $\mathrm{ScN}$, Akiyama et al. (2008) found that the piezoelectric response of AlN could be increased by $\sim 400 \%$ at a $43 \% \mathrm{ScN}$ concentration [11]. The microscopic origin of this effect was later studied by Tasnádi et al. [14], and they found that the effect in the $\mathrm{Sc}_{1-x} \mathrm{Al}_{x} \mathrm{~N}$ alloy is caused by the phase competition between the parent wurtzite phase of AlN and the layered hexagonal phase of ScN. The two phases are very similar, (see Fig.1.1) the only differences are that in the layered hexagonal phase the $\mathrm{Sc}$ and $\mathrm{N}$ ions are in the same plane, $u=0.5$, and the in plane distances are larger with the layers are more closely packed, i.e., a lower $c / a$-ratio. Thus, alloying $\mathrm{AlN}$ with $\mathrm{ScN}$ can both weaken the resistance to deformation of the parent wurtzite phase and increase the polarization change of such deformations. Tasnádi et al. also predict that the effect could be improved even more at higher $\mathrm{ScN}$ content, to a maximum at $50 \% \mathrm{ScN}$.

The discovery of the giant increase of the piezoelectric response and the fundamental explanation of the effect in ScAlN attracted our attention to the neighboring elements in the periodic table, the group 3 layered hexagonal and group 13 wurtzite nitrides. However, not all combinations provide an as large improvement of the piezoelectric response as ScAlN. The combinations which show the greatest improvement, and a reason behind their success is presented in Paper II. Although most of the research into the piezoelectric nitrides is currently focused on ScAlN, attention is starting to spread to the neighboring nitrides [15-17].

\subsection{Hard coatings}

Hard coatings have been used for many decades to increase the lifetime of tools, such as cutting tools, drill heads etc. $[18,19]$. One of the most successful hard coatings is TiN. Cubic TiN (c-TiN) is a hard material with a $\mathrm{NaCl}$ (B1) structure, see Fig.1.2. Objects coated by TiN have a characteristic gold colored surface. 


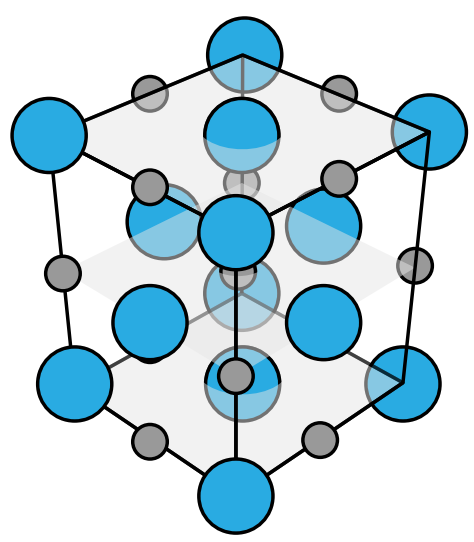

Figure 1.2. The cubic $\mathrm{NaCl}$ crystal structure of TiN.

Alloying TiN with AlN, has been found to improve both hardness [20-25] and oxidation resistance [26-29] of the film. This makes it possible to use it in a wide range of applications, e.g. high-speed cutting tools [18] and bio-implant coatings [19].

$\mathrm{Ti}_{1-x} \mathrm{Al}_{x} \mathrm{~N}$ is a metastable alloy which can be synthesized using PVD, allowing the growth of the film to occur at low temperatures so that there is no bulk diffusion, and only very limited surface diffusion. However, when it is subjected to high temperatures, such as during cutting operations, bulk diffusion is activated and the film starts to decompose into c-TiN and either c-AlN or wurtzite AlN (w-AlN) [30-35]. This is part of the reason behind the improved wear resistance observed experimentally $[30,31,33,34]$ and the interest in theoretical investigations [30, $34,36-38]$.

The atomic level dynamics behind the growth of $\mathrm{Ti}_{1-x} \mathrm{Al}_{x} \mathrm{~N}$ is very important to understand the micro- and nano-structural evolution of the film. However, it is difficult to investigate this experimentally, due to the very short time scales involved in surface diffusion events. Instead, it possible to use theoretical calculations based on first-principles and transition state theory (TST) [39, 40]. This method has been used to gain valuable knowledge about the surface kinetics of elemental metals [4, 41, 42], $\mathrm{TiC}[43,44]$ and the parent compounds TiN [5, 44-47] and $\mathrm{AlN}$ [48]. Modeling the surface kinetics for an alloy like $\mathrm{Ti}_{1-x} \mathrm{Al}_{x} \mathrm{~N}$, however, is more complex because of configurational disorder effects. ${ }^{2}$ It is therefore important to study the effects which the added $\mathrm{Al}$ has on the TiN surface energetics, by investigating the fully mixed $\mathrm{Ti}_{1-x} \mathrm{Al}_{x} \mathrm{~N}$, as we do in Paper IV, and in the much more dilute case where the local effects are much more distinguishable as we do Papers III.

\footnotetext{
${ }^{2}$ The problem with configurational disorder is further discussed in Sec. 2.3.
} 


\section{CHAPTER 2}

Methods

The main methods used in this work is described in this chapter. The first section describes the methods used to model atoms and electrons. The second section deals with the problem how to accurately model random alloys. The third section treats the methods necessary for modeling piezoelectric and elastic properties. The final section goes through the basics of diffusion and the methods used to study it.

\subsection{Modeling atomic and electronic structure}

The main reason for why we are able to do predictions of material properties directly from theory is the high accuracy of quantum mechanics in the description of atomic nuclei and electrons. Within quantum mechanics, we can describe a system of $N$ nuclei and $n$ electrons using a wave function $\Psi$,

$$
\Psi=\Psi\left(\mathbf{r}_{1}, \mathbf{r}_{2}, \ldots, \mathbf{r}_{n}, \sigma_{1}, \sigma_{2}, \ldots, \sigma_{n}, \mathbf{R}_{1}, \mathbf{R}_{2}, \ldots, \mathbf{R}_{N}, t\right)=\Psi(\overline{\mathbf{r}}, \bar{\sigma}, \overline{\mathbf{R}}, t)
$$

which is a function of the positions of the electrons $\mathbf{r}_{i}$, electron spins $\sigma_{i}$, nuclei positions $\mathbf{R}_{I}$, and time. The wave function is the solution to the Schrödinger equation, which determine the time evolution of the system. In atomic units the Schrödinger equation is

$$
i \frac{\partial \Psi}{\partial t}=H \Psi
$$

where $H$ is the Hamiltonian. In a system of nuclei and electrons without an external potential, the Hamiltonian is 


$$
\begin{array}{r}
H=-\frac{1}{2} \sum_{i=1}^{n} \nabla_{i}^{2}-\frac{1}{2} \sum_{I=1}^{N} \frac{1}{M_{I}} \nabla_{I}^{2}-\sum_{i, I} \frac{Z_{I}}{\left|\mathbf{r}_{i}-\mathbf{R}_{I}\right|} \\
+\frac{1}{2} \sum_{i \neq j} \frac{1}{\left|\mathbf{r}_{i}-\mathbf{r}_{j}\right|}+\frac{1}{2} \sum_{I \neq J} \frac{Z_{I} Z_{J}}{\left|\mathbf{R}_{I}-\mathbf{R}_{J}\right|} .
\end{array}
$$

The terms in the Hamiltonian correspond to, from left to right, the kinetic energy of the electrons, the kinetic energy of the nuclei, the potential energy of the electron-nucleus interaction, the electron-electron interaction, and the nucleusnucleus interaction.

If there is no specific time dependence in the Hamiltonian, it is possible to separate Eq. (2.2) in a time dependent and a spatial part. In many cases it is sufficient to look at the time independent part of the Schrödinger equation,

$$
H \Phi(\overline{\mathbf{r}}, \bar{\sigma}, \overline{\mathbf{R}})=E \Phi(\overline{\mathbf{r}}, \bar{\sigma}, \overline{\mathbf{R}})
$$

where $\Psi(\overline{\mathbf{r}}, \bar{\sigma}, \overline{\mathbf{R}})$ is the eigenfunction solution with the corresponding total energy eigenvalue E. Although Eq. (2.5) looks simple, solving it for systems with more than one hydrogen atom (two particles) quickly increases the difficulty.

It is worth to point out that we actually do not solve the Schrödinger equation in the codes we use to perform the calculations. Instead, a scalar-relativistic version of the Dirac equation is used. The fundamental points are, however, not different from when using the Schrödinger equation and the notation also become less cumbersome.

The Born-Oppenheimer approximation [49] is a very useful first approximation to reduce the number of particles considered in each calculation step. This is possible because the mass of the nuclei and the electrons differ by several orders of magnitude. Therefore, it is most of the times possible to deal first with electrons in a constant potential field caused by the nuclei. The kinetic effects on the nuclei can then be dealt with in a second step.

The problem with too many variables can be simplified in bulk materials with a second useful simplification, available because of the Bloch-theorem [50]. This allows us to use the periodicity of the crystal, and calculate only the unitcell of the crystal structure instead of every electron and nucleus in a macroscopic crystal. The wave function solutions to the Shrödinger equation then takes the form

$$
\phi_{n \mathbf{k}}(\overline{\mathbf{r}})=e^{i \mathbf{k} \cdot \mathbf{r}} u_{n \mathbf{k}}(\overline{\mathbf{r}}),
$$

where $n$ is the quantum number, $\mathbf{k}$ is a reciprocal vector, $u_{n \mathbf{k}}(\overline{\mathbf{r}})$ is a function with the periodicity of the lattice, and $e^{i \mathbf{k} \cdot \mathbf{r}}$ describes a plane wave. Although the problem at this point has been greatly simplified, there is so far no loss in accuracy. However, solving a system with more than a few electrons by solving the Schrödinger equation directly still poses a near impossible challenge. A this point, something more is needed to be done. This is where density functional theory comes in. 


\subsection{Density functional theory}

The basic idea of density functional theory is to use the electron density $n(r)$ as the basic variable instead of the wave function. This simplifies the problem of working with $3 n$ spatial variables for a $n$-electron problem to only 3 . The first attempts to use density functionals was in 1927 with the Thomas-Fermi theory $[51,52]$. However, the theory could not reproduce bonding between atoms, so it was generally discarded as a non-practical approach for many years.

\subsubsection{Hohenberg-Kohn}

It took until 1964, when Hohenberg and Kohn published their paper on inhomogeneous electron gas [53], for density functional theory to emerge in its modern form. The two theorems are stated in the words of Martin [54] as follows:

Theorem 1 For any system of interacting particles in an external potential $V_{\text {ext }}(\mathbf{r})$, the potential $V_{\text {ext }}(\mathbf{r})$ is determined uniquely, except for a constant, by the ground state particle density $n_{0}(\mathbf{r})$.

Theorem $2 A$ universal functional for the energy $E[n]$ in terms of the density $n(\mathbf{r})$ can be defined, valid for any external potential $V_{\text {ext }}(\mathbf{r})$. For any particular $V_{\text {ext }}(\mathbf{r})$, the exact ground state energy of the system is the global minimum value of this functional, and the density $n(\mathbf{r})$ that minimizes the functional is the exact ground state density $n_{0}(\mathbf{r})$.

The first theorem has the consequence that all properties of a system are completely determined by the ground state density $n_{0}(\mathbf{r})$, and the second that $E[n]$ alone is enough to determine the exact ground state energy and density. The general form of the functional is

$$
E[n]=T[n]+E_{\text {int }}[n]+\int d^{3} r V_{e x t}(\mathbf{r}) n(\mathbf{r})+E_{I I},
$$

where the terms on the right side of the equation represent the kinetic energy of the electrons, the electron-electron interaction energy, the interaction energy with an external potential in the form of Coulomb interaction with the nuclei, and the interaction energy between the nuclei. Although the form of these functionals is not known, a practical scheme to solve this problem was proposed already the year after.

\subsubsection{Kohn-Sham}

Kohn and Sham suggested in 1965 [55] that the real system with interacting particles should be replaced with a system of noninteracting particles. This is done by replacing the purely external potential with an effective potential $V_{\text {eff }}$. The single particles interacting with the effective potential is then described by the single particle wave function $\psi_{j}$, which is found by solving the single-particle equation $[56]$ 


$$
\left(-\frac{1}{2} \nabla^{2}+V_{e f f}(\mathbf{r})-\epsilon_{j}\right) \psi_{j}(\mathbf{r})=0
$$

where $\epsilon_{j}$ is the eigenvalue of the non-interacting single particle, and the effective potential is given by

$$
V_{e f f}(\mathbf{r})=V_{e x t}(\mathbf{r})+\int \frac{n\left(\mathbf{r}^{\prime}\right)}{\left|\mathbf{r}-\mathbf{r}^{\prime}\right|} d \mathbf{r}^{\prime}+V_{x c}(\mathbf{r}),
$$

where

$$
V_{x c}=\frac{\delta E_{x c}[n(\mathbf{r})]}{\delta n(\mathbf{r})}
$$

The electron density in a system with $N$ electrons is then calculated using the wave functions according to

$$
n(\mathbf{r})=\sum_{j=1}^{N}\left|\psi_{j}(\mathbf{r})\right|^{2} .
$$

The total energy is given by the Kohn-Sham total energy functional

$$
E_{K S}[n]=T_{s}[n]+\int d \mathbf{r} V_{e x t}(\mathbf{r}) n(\mathbf{r})+E_{\text {Hartree }}[n]+E_{I I}+E_{x c}[n] .
$$

Here, $V_{\text {ext }}(\mathbf{r})$ is the external potential due to the nuclei and any other external fields (assumed to be independent of spin), $E_{I I}$ is the interaction between the nuclei, and $E_{\text {Hartree }}$ is the classical Coulomb interaction energy of the electron density with itself given by

$$
E_{\text {Hartree }}=\frac{1}{2} \int d^{3} r d^{3} r^{\prime} \frac{n(\mathbf{r}) n\left(\mathbf{r}^{\prime}\right)}{\left|\mathbf{r}-\mathbf{r}^{\prime}\right|} .
$$

Although the Kohn-Sham total energy functional is exact in the form presented in Eq. (2.12), the exchange-correlation term $E_{x c}[n]$, where all many-body effects are included, pose a problem because there is no universal form for it. However, effects of this term is in general small compared to the other terms which are evaluated almost exactly. There also exist approximate functionals which capture many-body effects with good accuracy, e.g., the local-density approximation and the generalized gradient approximation which are presented below.

\subsubsection{Local-density approximation}

The local-density approximation (LDA) was first suggested in the original paper by Kohn and Sham [55], and later extended to also include spin with the local spin density approximation (LSDA) [57]. The basic idea comes from the observation that exchange-correlation effects are to a large extent local in character. Therefore, they proposed to calculate the exchange-correlation energy $E_{x c}^{L D A}[n]$ with a simple 
integral over all space, where the exchange-correlation energy density is assumed to be the same as in homogeneous electron gas with that density $\epsilon_{x c}^{\text {hom }}[n(\mathbf{r})]$,

$$
E_{x c}^{L D A}[n]=\int d^{3} n(\mathbf{r}) \epsilon_{x c}^{h o m}[n(\mathbf{r})]
$$

The homogeneous electron gas has been studied to great accuracy using quantum Monte Carlo calculations [58], creating a set of data for different densities. The densities between the points in the data set has then been interpolated [59, $60]$.

The initial expectations about the performance of LDA was that it would only work well in the limit where the density varies slowly. However, LDA turned out to produce useful results also for more complex systems [56]. This is because of the exchange-correlation hole, many-body effects depleting the electron charge closest to the electrons. Furthermore, LDA also obeys the sum rule, that if we integrate the entire exchange-correlation hole we will end up with exactly the charge of one electron, even if the shape of the hole is not entirely correct. The reason why the shape of the hole does not matter that much is because only the spherical average of the exchange-correlation hole enters the energy, and this is well reproduced by LDA.

To summarize the performance of LDA, it works well for covalent-, metalic-, and ionictype bonds, even though it generally tends to overbind slightly. Although the overbinding causes gives lattice parameters which are lower than those experimentally obtained, trends in lattice parameters are well reproduced. However, for long range interactions such as Van der Waal's interactions LDA does not work.

\subsubsection{Generalized gradient approximation}

The generalized gradient approximation (GGA) improves the LDA by including the absolute value of the gradient of the density with the value of $n$ at each point. There are a number of implementations to introduce the gradient corrections [6164], which still force the system to behave correctly in important limiting cases [54].

The work in this thesis has been performed using the implementation by Perdew, Burke, and Ernzerhof (PBE-GGA) [63, 65]. Although it tends to underbind and provide slightly larger lattice constants than experiment, PBE-GGA is better at providing reasonable geometries for the nitride systems investigated in this thesis, as compared to the LDA.

\subsubsection{Beyond LDA and GGA}

The limitations in LDA and GGA has called for many new functionals, e.g., $\mathrm{LDA}+\mathrm{U}[66-68]$ and hybrid functionals such as B3LYP [61, 69], improving features such as bandgaps and long range interactions.

Another issue is the accuracy of adsorption and surface energies obtained by DFT has received some criticism lately by Schimka et al. [70], pointing out limitations in commonly used pseudopotentials. The main problem they address is 
for configurations where van der Waals forces are important contributors, such as admolecules. However, there is no evidence this would be an alarming issue for single adatoms. For a single adatom, the van der Waals force contribution to the adsorption energy is much less than the short range binding to the nearest surface atoms.

A general issue with most post LDA and GGA exchange-correlation methods is that the increased accuracy also greatly increases the computational time, usually many times more the LDA and GGA computational time. Therefore, using one of these improved functionals for problems where LDA and GGA anyway performs well is not economical in terms of computer resources.

\subsubsection{Basis sets}

Basis sets are used when solving the Kohn-Sham equations numerically, there they are used to expand the wave function in Eq. (2.1). A natural choice is to use plane waves as the basis set, because they work well with tools related to Fourier transforms. However, the rapid variations in the wave functions and effective potentials close to the nuclei are problematic to describe with plane waves. A huge set of plane waves with high energy cutoff would be needed in order to simultaneously describe the environment close to the nuclei with high kinetic energies as well as the smother region between the atoms. Using pseudopotentials is a way to get around this problem.

The basic idea of the pseudopotential is to replace the problematic inner region of the atom (the nucleus and the inner core electrons ${ }^{1}$ ) with an effective ionic potential acting on the valence electrons. This approach has several benefits; the basis set size is reduced, the number of electrons is reduced, and it is possible to include more effects such as relativistic effects if they are not already included.

Although there are many different kinds of pseudopotentials which can be used to simplify the calculations, the general approach is to construct the potential in a way that it reproduces the scattering properties of the core region within a certain cutoff radius and the behavior of the valence wave functions and the effective potentials outside it.

Soft pseudopotentials employ a larger cutoff radius, this does provide faster converging calculations, however, it also makes the less transferable. Not being able to transfer a pseudopotential between crystal structure or chemical environments means that one has to go through the extensive work of parameterize a new one. An approach to solve this problem with tranferability is to use ultrasoft pseudopotentials [71], which was used in the calculations in Paper II. In this approach, this is accomplished by introducing a generalized orthonormality condition, and making sure that the full electronic charge is recovered, by augmenting the electron density in the core regions.

The calculation in Paper I, III, and IV, were all performed using projector augmented wave (PAW) approach $[72,73]$. Although the PAW method is similar to the ultrasoft approach, it retains the entire set of all-electron core functions

\footnotetext{
${ }^{1}$ Including the inner core electrons is generally not a problem since they are tightly bound to in the core.
} 
along with the smooth parts of the valence functions. This makes it possible to reconstruct all electron wave functions from the pseudo-wavefunctions. The two approaches are similar in accuracy, however, the PAW is more reliable for magnetic systems [73].

\subsection{Modeling random alloys}

A review on the subject theoretical modeling of random alloys is covered in Ref. [74]. The following section is inspired by that work.

\section{Configurational disorder}

One of the most challenging problems with modeling alloys like $\operatorname{Ti}_{1-x} \mathrm{Al}_{x} \mathrm{~N}$ is the configurational disorder, where the $\mathrm{Ti}$ and $\mathrm{Al}$ atoms are positioned more or less randomly at cation sites in the lattice. In random alloys, there is no longrange order, although short-range order (SRO) can exist, therefore, the Blochs theorem (Sec. 2.1) is no longer valid. Thus, the crystal unit cell is not enough to simulate the material's properties. In practice, this can be modeled with a supercell of several unitcells, but the larger the system, the more computer resources are required. Therefore, we want to use an as small simulation box as possible which still represent the properties of the random alloy. In a formal sense, a random alloy can be defined as a system with an atomic configuration in the limit $V / T \rightarrow 0$, where $V$ is the strongest effective configurational interaction in the system and $T$ is the temperature.

(a)

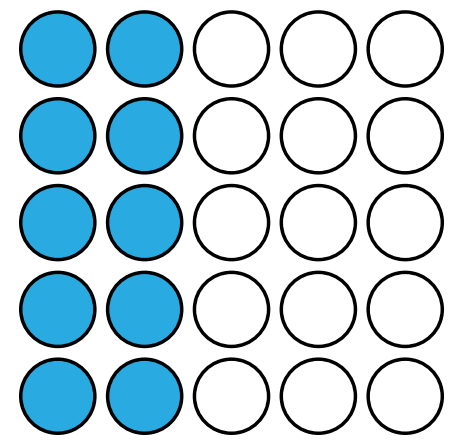

(b)

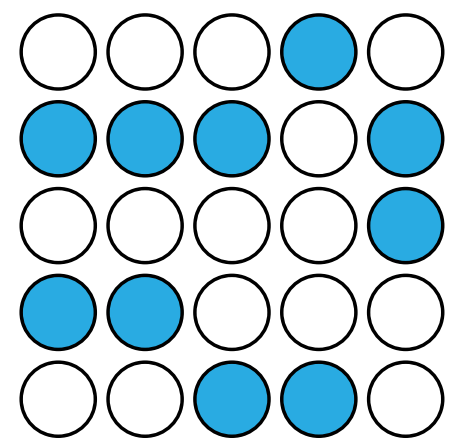

Figure 2.1. Simple illustration of the configurational problem. System (a) and system (b) has the same composition, but different configurations of atoms.

The importance of using a random configuration is illustrated in Fig. 2.1, where (a) and (b) are cells representing two possible outcome of randomly generated configurations of colored and white atoms. In this example it might appear intuitive which one would best represent a random alloy when periodic boundaries are applied, whereas the situation is actually very complex. Should we continue to 
randomly place atoms and by increasing the size too infinity, then it would practically not matter which supercell is used. Of course, an extremely large supercell is not useful from a calculation point of view. Instead, it is advisable to use the special quasi-random structure (SQS) models [75].

\subsubsection{Special quasi-random structure model}

To understand how large such a cell should be and what conditions it should satisfy it is necessary to understand the concept of cluster expansion of the configurational part of the total energy. The formalism necessary for this was developed by Sanchez, Ducastelle, and Gratias [76, 77].

For a simple case of a binary alloy, $\mathrm{A}_{x} \mathrm{~B}_{1-x}$, we can use spin variables $\sigma_{i}$ to describe the atomic configuration. $\sigma_{i}$ takes on the value +1 if the site is occupied by an $\mathrm{A}$ atom and -1 if its occupied by a $\mathrm{B}$ atom. In a crystal with $N$ sites, the vector containing all the spin variables is then $\boldsymbol{\sigma}=\left\{\sigma_{1}, \sigma_{2}, \sigma_{3}, \ldots, \sigma_{N}\right\}$. A characteristic function $\Phi_{f}^{(n)}(\boldsymbol{\sigma})$ can be defined for a given $n$-site cluster, which is given by the product of the spin variables $\sigma_{i}$ in the cluster $\alpha$,

$$
\Phi_{\alpha}^{(n)}(\boldsymbol{\sigma})=\prod_{i \in \alpha} \sigma_{i}
$$

The scalar product of two such functions at any point $i$ form a complete and orthogonal set with the inner product

$$
\left\langle\Phi_{\alpha}^{(n)}(\boldsymbol{\sigma}), \Phi_{\beta}^{(n)}(\boldsymbol{\sigma})\right\rangle=\frac{1}{2^{N}} \sum_{\boldsymbol{\sigma}} \Phi_{\alpha}^{(n)}(\boldsymbol{\sigma}) \Phi_{\beta}^{(n)}(\boldsymbol{\sigma})=\delta_{\alpha, \beta},
$$

where the summation goes over all possible configurations $\boldsymbol{\sigma}$. This means that if two clusters differ by at least one site, then the function equals to 0 , and 1 if they are the same. This means that any function of the configuration

$$
F(\boldsymbol{\sigma})=\sum_{\alpha} F_{\alpha}^{(n)} \Phi_{\alpha}^{(n)}(\boldsymbol{\sigma})
$$

can be expanded in this basis set. The expansion coefficient in this equation are purely the projections

$$
F_{\alpha}^{(n)}=\left\langle F(\boldsymbol{\sigma}), \Phi_{\alpha}^{(n)}(\boldsymbol{\sigma})\right\rangle
$$

on the basis function.

In the case of total energy $E_{\text {tot }}$ of an alloy configuration, the expansion coefficients are called effective cluster interactions $V_{\alpha}^{(n)}$, given by

$$
V_{\alpha}^{(n)}=\left\langle E_{t o t}(\boldsymbol{\sigma}), \Phi_{\alpha}^{(n)}(\boldsymbol{\sigma})\right\rangle
$$

If we introduce the definition of the statistical cluster correlation function $\xi_{f}^{(n)}$ for a given configuration $\boldsymbol{\sigma}$ as the average of the symmetrically identical cluster functions, 


$$
\xi_{f}^{(n)}(\boldsymbol{\sigma})=\left\langle\Phi_{f}^{(n)}\right\rangle=\frac{1}{m_{f}^{(n)}} \sum_{\forall \alpha \in f} \Phi_{\alpha}(\boldsymbol{\sigma}) .
$$

where $m_{f}^{(n)}$ is a normalization factor. The total energy can then be given in terms of symmetrically non-equivalent figures using the expression

$$
E_{t o t}=\sum_{f} V_{f}^{(n)} m_{f}^{(n)} \xi_{f}^{(n)} .
$$

Often, the term cluster is used instead of figure, which can cause some confusion.

From Eq. (2.21), it is possible to draw conclusions about how a supercell should be constructed in order to mimic a truly random structure. First of all, it is clear that the only clusters that can contribute to the alloy energetics are the ones where $V_{f}^{(n)} \neq 0$. This means that only their correlation functions are important. Therefore, an SQS supercell should be constructed so that $\xi_{f}^{(n)}=0$, as it is in a random structure, for as many of these clusters as possible. ${ }^{2}$ Although a finite supercell will not be able to do this for all distance cluster, the interactions corresponding to pair clusters at short distances are generally more important than those between more distant neighbors. Therefore, an SQS will best represent the random alloy if it is generated with as many of the first few nearest neighbor correlation functions as possible equal to zero.

\subsubsection{Limitations of the special quasi-random structure model}

Although the SQS models possible to compute with today's supercomputers are excellent at reproducing the total energy of a random alloy, there are limitations to the model. Using a perfectly random alloy is in it self an approximation since real alloys tend to have some degree of clustering or ordering at short-range scales. However, it is a suitable unbiased starting point when approaching unknown alloys.

One of the limitation with the SQS method is calculating tensorial properties such as elastic constants. A recent study by Tasnádi, Odén, and Abrikosov [78] pointed out a problem with obtaining tensor properties from SQS models and proposed a solution to the problem. The problem with the SQS approach is that it is not designed to preserve the point group symmetry of an alloy, and thus the tensorial properties. This has not been taken into account when generating the SQS structures used in Paper II. However, the same SQS structure was used when comparing the elastic constants of the different alloys. Therefore, although the results can differ somewhat from experimental values because of the choice of SQS, any difference caused by the SQS will be the same for all. Thus, trends should not be strongly influenced.

\footnotetext{
${ }^{2}$ The SQS approach is not limited to random alloys, where $\xi_{f}^{(n)}=0$. It can also be extended to model clustering or ordering by specifying $\xi_{f}^{(n)}$ other than zero.
} 
Another limitation with the SQS method is related to the surface diffusion. The problem can be illustrated with Fig. 2.1 (b), by considering an alternative representation. In this case, the circles represent binding sites on a surface, where a fictive adatom is only allowed to move between white sites and not diagonally, to keep it simple. Then it is clear that using this with periodic boundary conditions will promote diffusion "highways", which in this case means that all diffusion is along the left and right direction while up and down is completely blocked. Although this example is exaggerated, this is a limitation of the SQS approach. This is one of the problems discussed in Paper IV, where we investigate the surface of an SQS model, and also a reason behind why it is important to consider the dilute limit of surface atom substitutions which is done in Paper III.

\subsection{Phase stability}

If a material is said to be stable, it means that the material is in thermodynamic equilibrium, in a global minimum in the Gibb's free energy $G$, which is defined as [79]

$$
G=H-T S,
$$

where $T$ is the temperature, $S$ is the entropy, and the enthalpy $H$ is given by

$$
H=E+P V,
$$

where $E$ is the total energy, $P$ is the pressure, and $V$ is the volume.
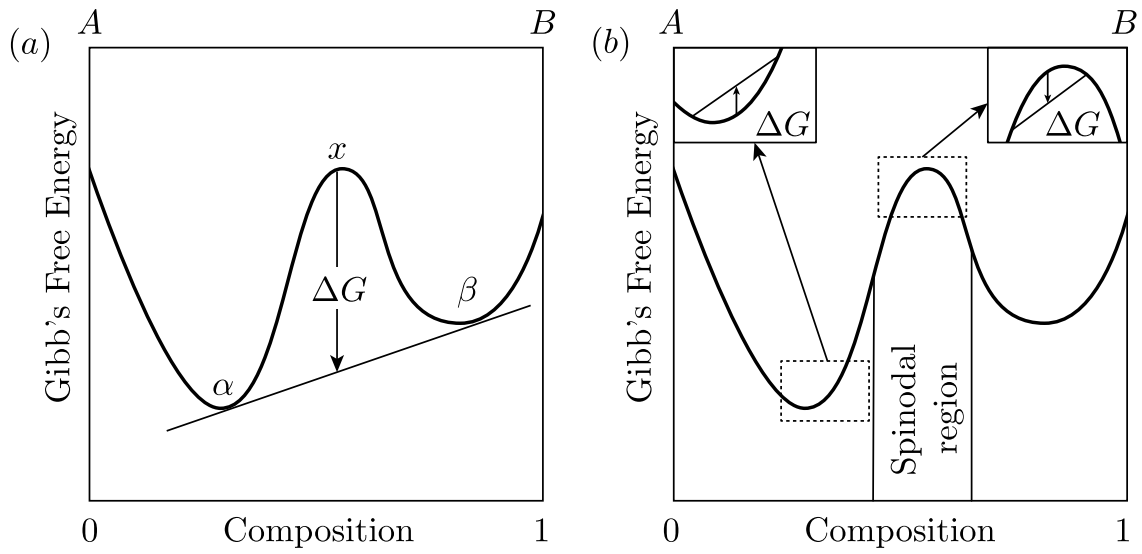

Figure 2.2. Gibb's free energy for a fictive material. (a) Reduction of the free energy with $\Delta G$ when the system with composition $x$ reaches equilibrium. (b) Free energy differences caused by small fluctuations in the local compositon.

Metastable materials are also important, because they potentially possess better material properties than the stable state. A metastable material is in a local 
minimum in $G$, this phase is stable enough to withstand some variations in external conditions and/or temperature induced fluctuations, before transitioning to a more stable state due to the thermodynamic driving force to always reduce the Gibb's free energy.

A common way to improve the properties of a material is to alloy it with different concentrations $x$ of an other element. However, not all concentrations produce a stable alloy. It is therefore advantageous to determine the stability of a mixture by calculating the mixing free energy $\Delta G_{m i x}$, according to

$$
\Delta G_{m i x}=\Delta H_{m i x}-T \Delta S_{m i x}
$$

where the contribution comes from the mixing enthalpy $\Delta H_{m i x}$ and the mixing entropy $\Delta S_{m i x}$.

The Gibb's free energy with respect to concentration for a fictive binary system is presented in Fig. 2.2. In part (a) of the figure is an illustration of the ground state configuration of a mixture with the concentration $x$. From a homogeneous mixture at $x$, the material will reduce $\Delta G_{m i x}$ by decomposing and forming phases with the concentration $\alpha$ and $\beta$.

The mechanism behind the decomposition is related to the second derivative of the free energy. If $d^{2} G / d x^{2}>0$, then small fluctuations will lead to an increase in the free energy. The decomposition is therefore driven by nucleation and growth and limited by an interface related nucleation barrier. Due to this barrier the system can be considered as metastable in the thermodynamic sense. In the opposite case when $d^{2} G / d x^{2}<0$, within the spinodal region, small fluctuations lead to a decrease in the free energy. The mechanism behind the decomposition in this region is called spinodal decomposition, and is not required to overcome a nucleation barrier. Although an alloy within this concentration range is much more sensitive to decomposition, this does not necessary mean that it is impossible to synthesize it. During thin film growth it is possible to keep temperatures low enough to stop the bulk diffusion and, thus, the decomposition, while appreciating the possible surface-initiated decomposition due to more easily activated surface diffusion. Such alloys, although unstable in the thermodynamic sense, can be metastable due to kinetic limitations.

\subsubsection{Approximate calculations for non-equilibrium condi- tions}

The temperature dependence of Gibb's free energy is the largest challenge for first-principles phase stability calculation. However, in many cases, important information can be gained from the mixing enthalpy alone, which, for zero pressure, can be calculated from total energies calculations at the equilibrium volumes.

Under the low temperature non-equilibrium conditions of PVD nitride thin film growth, long range diffusion is quenched and phase separation can often not occur. Instead, it is more useful to study possible competing alloy phases, e.g., wurtzite or rock salt, at each investigated composition. In such cases, the configurational entropy for a binary or quasi-binary solution, reasonably approximated with 


$$
S=-k_{b}(x \ln (x)+(1-x) \ln (1-x)),
$$

where $k_{b}$ is the Boltzmann constant, would be the same for all disordered alloy phases and, therefore, not influence the free energy balance.

The temperature dependent vibrational free energy can of course be different between different crystal structures. However, as a typical PVD process for nitrides is performed at a third of the melting temperature, the vibrational contributions are at least not dominating the free energy balance.

If the entropy addition to the free energy differences can be approximated as zero, the remaining important contribution comes from mixing enthalpy of each respective alloy phase. These can be calculated using DFT and the SQS formalism described previously. Of interest to this work is, for example, for which compositions that the wurtzite phase is retained when alloying a wurtzite group 13 nitride with a group three nitride, such as AlN with YN, which is studied in Paper. I. The crossing points of the mixing enthalpy curves for the competing structures are good guidelines for maximum solubility limits under PVD conditions.

In addition to the bulk thermodynamics, the surface energies of each particular phase are also relevant when predicting the structures of thin films. Nonetheless, as important is a direct consideration of the growth kinetics, as will be discussed later in Sec. 2.6.

\subsection{Modeling elastic and piezoelectric properties}

The piezoelectric properties of a material describe how much electric charge is accumulated when stress is applied to it. The first step in the process of calculating the piezoelectric properties from a theoretical approach is to calculate the elastic properties of the material.

\subsubsection{Elastic properties}

The elastic properties of a material is a key component in describing its mechanical properties, and it is especially important for thin films, because the stress/strain relation between the substrate and the film determines whether or not the film will stick to the substrate. If the lattice parameters differ too much between the film and the substrate and the film is not elastic enough, then the film will not be able to stick to the substrate. Elasticity is also an important property relating to piezoelectric response, which will be covered in section 2.5.

The elastic properties of a material are described by the relation between stress $\sigma_{\alpha \beta}$ and strain $\epsilon_{\gamma \delta}$. The relation between the two, and a method to calculate it using theoretical modeling, is found following Finnis [80]. Using this approach, we start with the matrix representation of homogeneous strain

$$
\epsilon=\left(\begin{array}{lll}
\epsilon_{11} & \epsilon_{12} & \epsilon_{13} \\
\epsilon_{21} & \epsilon_{22} & \epsilon_{23} \\
\epsilon_{31} & \epsilon_{32} & \epsilon_{33}
\end{array}\right)
$$


Assuming that the strain moves a point from $\mathbf{r}=\left(x_{1}, x_{2}, x_{3}\right)$ to the point $\mathbf{r}+\mathbf{u}$, then the elements of the matrix are defined as

$$
\epsilon_{\alpha \beta}=\frac{1}{2}\left(\frac{\partial u_{\beta}}{\partial x_{\alpha}}+\frac{\partial u_{\alpha}}{\partial x_{\beta}}\right) .
$$

The stress $\sigma_{\alpha \beta}$ is then connected via the elastic constants $C_{\alpha \beta \gamma \delta}$ as

$$
\sigma_{\alpha \beta}=\sum_{\gamma \delta} C_{\alpha \beta \gamma \delta} \epsilon_{\gamma \delta}
$$

The symmetry allows the matrices to be simplified using Voigt notation as

$$
\left(\begin{array}{l}
\epsilon_{1} \\
\epsilon_{2} \\
\epsilon_{3} \\
\epsilon_{4} \\
\epsilon_{5} \\
\epsilon_{6}
\end{array}\right)=\left(\begin{array}{c}
\epsilon_{11} \\
\epsilon_{22} \\
\epsilon_{33} \\
2 \epsilon_{23} \\
2 \epsilon_{13} \\
2 \epsilon_{12}
\end{array}\right)
$$

and

$$
\left(\begin{array}{l}
\sigma_{1} \\
\sigma_{2} \\
\sigma_{3} \\
\sigma_{4} \\
\sigma_{5} \\
\sigma_{6}
\end{array}\right)=\left(\begin{array}{l}
\sigma_{11} \\
\sigma_{22} \\
\sigma_{33} \\
\sigma_{23} \\
\sigma_{13} \\
\sigma_{12}
\end{array}\right)
$$

This makes it possible to also reduce the elastic constant matrix to a $6 \times 6$ matrix $C_{i j}$ and simplify Eq.(2.28) to

$$
\sigma_{i}=\sum_{j} C_{i j} \epsilon_{j}
$$

$C_{i j}$ can be more or less simple, because many of the components are zero due to point symmetry in the structure. For cubic crystals there are only 3 independent elastic constants $\left(C_{11}, C_{12}\right.$, and $\left.C_{44}\right)$, and for hexagonal crystals there are $5\left(C_{11}\right.$, $C_{12}, C_{13}, C_{33}$, and $\left.C_{44}\right)$. The elastic constant matrix for hexagonal crystals in full is [81]

$$
C_{i j}=\left(\begin{array}{cccccc}
C_{11} & C_{12} & C_{13} & 0 & 0 & 0 \\
C_{12} & C_{11} & C_{13} & 0 & 0 & 0 \\
C_{13} & C_{13} & C_{33} & 0 & 0 & 0 \\
0 & 0 & 0 & C_{44} & 0 & 0 \\
0 & 0 & 0 & 0 & C_{44} & 0 \\
0 & 0 & 0 & 0 & 0 & \frac{1}{2}\left(C_{11}-C_{12}\right)
\end{array}\right)
$$


The five independent matrix elements for hexagonal crystals is calculated from of the elastic energy per unit volume $U$ which is defined as [80]

$$
U=\frac{1}{2} \sum_{i j} C_{i j} \epsilon_{i} \epsilon_{j} .
$$

The strain configurations $\epsilon=\left(\epsilon_{1}, \epsilon_{2}, \epsilon_{3}, \epsilon_{4}, \epsilon_{5}, \epsilon_{6}\right)$ and their corresponding elastic energy function that can be used to determine the five elastic constants are

$$
\begin{aligned}
\epsilon^{1}=(\delta, \delta, 0,0,0,0) \rightarrow U^{1} & =\left(C_{11}-C_{12}\right) \delta^{2}, \\
\epsilon^{2}=(\delta, \delta,-2 \delta, 0,0,0) \rightarrow U^{2} & =\left(C_{11}+C_{12}-4 C_{13}+2 C_{33}\right) \delta^{2}, \\
\epsilon^{3}=(0,0, \delta, 0,0,0) \rightarrow U^{3} & =\frac{1}{2} C_{33} \delta^{2}, \\
\epsilon^{4}=(0,0,0,0,0, \delta) \rightarrow U^{4} & =\frac{1}{4}\left(C_{11}-C_{12}\right) \delta^{2}, \\
\epsilon^{5}=(0,0,0, \delta, \delta, 0) \rightarrow U^{5} & =C_{44} \delta^{2},
\end{aligned}
$$

where $\delta$ is a set of small distortions of $\pm 2 \%$. The elastic constants can then be obtained by fitting a second order polynomial to the data set. Note that only $C_{33}$ and $C_{44}$ can be directly obtained from a single set of distortions, the others can only be found in combinations with other distortion sets. Of these two, $C_{33}$ is the most important in this work, since this is the elastic constant for the $c$-direction of the hexagonal crystal, which is the first part of determining the response of a piezoelectric wurtzite material.

\subsubsection{Piezoelectric properties}

There are four material coefficients which describe the piezoelectric properties of a material, $d_{i j}, e_{i j}, g_{i j}$, and $h_{i j}$. These connect stress $\sigma$ and strain $\epsilon$ to changes in the electrical and dielectric fields $E$ and $D$ and vice versa via the relations

$$
\begin{gathered}
d_{i j}=\left(\frac{\partial D_{i}}{\partial \sigma_{j}}\right)_{E}=\left(\frac{\partial \epsilon_{j}}{\partial E_{i}}\right)_{\sigma}, \\
e_{i j}=\left(\frac{\partial D_{i}}{\partial \epsilon_{j}}\right)_{E}=-\left(\frac{\partial \sigma_{j}}{\partial E_{i}}\right)_{\epsilon}, \\
g_{i j}=-\left(\frac{\partial E_{i}}{\partial \sigma_{j}}\right)_{D}=\left(\frac{\partial \epsilon_{j}}{\partial D_{i}}\right)_{\sigma}, \\
h_{i j}=-\left(\frac{\partial E_{i}}{\partial \epsilon_{j}}\right)_{D}=-\left(\frac{\partial \sigma_{j}}{\partial D_{i}}\right)_{\epsilon},
\end{gathered}
$$

where the first part of the equations correspond to the direct piezoelectric effect, and the second part correspond to the converse piezoelectric effect [82]. A helpful 
schematic overview of the relations between physical properties and the piezoelectric coefficients is presented in Fig. 2.3.

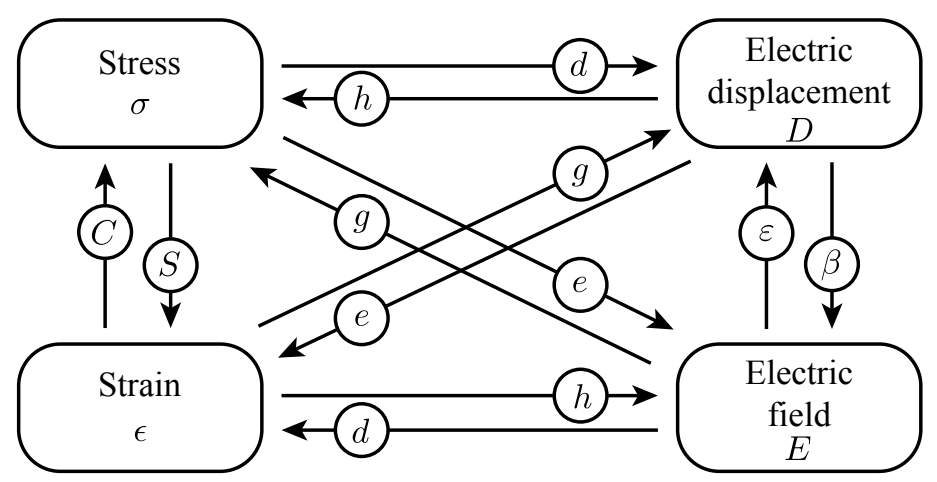

Figure 2.3. Schematic overview of the relations between piezoelectric coefficients, elastic constants, electric permittivity, stress, strain, electric displacement and electric field. Adapted from Ref.[82].

The piezoelectric coefficient $e$ can be calculated within DFT using the Berryphase approach suggested by Bernardini, Fiorentini, and Vanderbilt [83]. This approach focuses on investigating how the polarization in the material changes when strain is applied to it.

The total polarization $\mathbf{P}$ is given by

$$
\mathbf{P}=\mathbf{P}^{e q}+\delta \mathbf{P}
$$

where $\mathbf{P}^{e q}$ is the polarization of the equilibrium structure and $\delta \mathbf{P}$ is the piezoelectric polarization. The piezoelectric part is, in the linear regime, given by

$$
\delta P_{i}=\sum_{j} e_{i j} \epsilon_{j},
$$

where $\epsilon_{j}$ is the strain component.

For wurtzite thin film, the standard growth direction is the $c$-direction, and any distortions of the structure will be along this direction. Therefore, the focus is on $\delta P_{3}$ which is defined as

$$
\delta P_{3}=e_{33} \epsilon_{3}+e_{31}\left(\epsilon_{1}+\epsilon_{2}\right) .
$$

In this expression $\epsilon_{3}$ is the strain along the $c$-axis, and $\epsilon_{1}$ and $\epsilon_{2}$ are the in-plane strain.

The wurtzite structure has three independent components in the piezoelectric tensor, $e_{33}, e_{13}$, and $e_{15}$. The final component $e_{15}$ is related to shear strain which is not expected to be of great importance for a thin film, since it is so flat.

With $c_{0}$ and $a_{0}$ as the equilibrium lattice constants, the strain components in Eq.(2.45) can be replaced with 


$$
\epsilon_{3}=\frac{c-c_{0}}{c_{0}}
$$

and, assuming that $\epsilon_{1}$ and $\epsilon_{2}$ are isotropic,

$$
\epsilon_{1}=\epsilon_{2}=\frac{a-a_{0}}{a_{0}}
$$

so that the change in polarization can be be expressed as

$$
\delta P_{3}=\frac{\partial P_{3}}{\partial a}\left(a-a_{0}\right)+\frac{\partial P_{3}}{\partial c}\left(c-c_{0}\right)+\frac{\partial P_{3}}{\partial u}\left(u-u_{0}\right),
$$

where the third term takes into account changes in polarization due to internal relaxation changing the internal equilibrium parameter $u_{0}$.

When these derivatives are known, the piezoelectric coefficients can be calculated using [83]

$$
e_{33}=c_{0} \frac{\partial P_{3}}{\partial c}+\frac{4 e c_{0}}{\sqrt{3} a_{0}^{2}} Z^{*} \frac{d u}{d c}
$$

and

$$
e_{31}=\frac{a_{0}}{2} \frac{\partial P_{3}}{\partial a}+\frac{2 e}{\sqrt{3} a_{0}} Z^{*} \frac{d u}{d a},
$$

where the dynamical Born effective charge $Z^{*}$ is

$$
Z^{*}=\frac{\sqrt{3} a_{0}^{2}}{4 e} \frac{\partial P_{3}}{\partial u} .
$$

The first term in Eq. (2.49) and (2.50) corresponds to the clamped-ion term, which represent the effect of the strain on the electronic structure. The second term corresponds to effects of internal strain on the polarization. Note that $e$ without indices is the elementary charge.

Most of the parameters needed to calculate $e_{33}$ and $e_{13}$ are straight forward to calculate by distorting the structure. However, the polarization is more complex to derive.

\subsubsection{Berry-phase theory of polarization}

The difficulty calculating polarization comes from that it is not a bulk property, which means it is dependent on the shape and truncation of the sample [84]. A more complex approach is therefore needed.

The Berry-phase approach, as stated by Vanderbilt [85], calculates the polarization in a system using geometric quantum phases known as Berry phases. ${ }^{3}$ The total polarization is [85]

\footnotetext{
${ }^{3}$ Berry phases can be useful for calculating many other electronic properties other than polarization. For an extensive review of the subject see Ref. [86]
} 


$$
\mathbf{P}=\frac{e}{\Omega} \sum_{\tau} Z_{\tau} \mathbf{r}_{\tau}+\sum_{n \text { occ }} \mathbf{P}_{n}
$$

where $\Omega$ is the unitcell volume, $e$ is the elementary charge, $Z_{\tau}$ is the atomic number of the $\tau$-th nucleus, $\mathbf{r}_{\tau}$ its position. The firs part is the contribution from the nuclei and the second part comes from the spontaneous electronic polarization of the occupied valence bands.

With Berry phases $\phi, \mathbf{P}_{n}$ can be written as

$$
\mathbf{P}_{n}=-\frac{1}{2 \pi} \frac{e}{\Omega} \sum_{\alpha} \phi_{n, \alpha} \mathbf{R}_{\alpha},
$$

where $\mathbf{R}_{\alpha}$ is a real-space primitive lattice vector corresponding to the reciprocalspace primitive lattice vector $\mathbf{G}_{\alpha}$.

$$
\phi_{n, \alpha}=\Omega_{\mathrm{BZ}}^{-1} \int_{\mathrm{BZ}} \mathrm{d}^{3} k\left\langle u_{n \mathbf{k}}\left|-i \mathbf{G}_{\alpha} \cdot \nabla_{\mathbf{k}}\right| u_{n \mathbf{k}}\right\rangle,
$$

where $\Omega_{B Z}$ is the volume of the Brillouin zone (BZ), and $u_{n \mathbf{k}}=e^{i \mathbf{k} \cdot \mathbf{r}} \psi_{n \mathbf{k}}(\mathbf{r})$ is the cell-periodic Bloch functions.

For a full derivation of this approach see Refs. [83-86].

\subsubsection{Piezoelectric response $d$}

The piezoelectric coefficient $e_{i j}$ cannot be directly obtained experimentally, however, $d_{i j}$ can. Therefore, it is useful to convert $e_{i j}$ to the corresponding $d_{i j}$. The relation between the relevant components are given by [87]

$$
e_{31}=d_{31}\left(c_{11}+c_{12}\right)+d_{33} c_{13}
$$

and

$$
e_{33}=2 d_{31} c_{13}+d_{33} c_{33} .
$$

The origin of the enormous increase of the piezoelectric response in ScAlN, is due to an increase in the internal strain term of the $e_{33}$ coefficient and a decrease in elastic constant $C_{33}$.[14] With the addition that $C_{33}>C_{31}$ and $d_{33}>d_{31}$, the second term in Eq.(2.56) is much greater than the first term so that

$$
d_{33} \approx \frac{e_{33}}{c_{33}}
$$

is a good approximation of $d_{33} \cdot[14]$ The validity of this approximation depends on the device it is intended for. For devices where the piezoelectric response is due to bending of the material, the $e_{13}$ coefficient can be more important than when strain is applied only along the $c$-direction.

Nonetheless, primarily studying the three coefficients $C_{33}, e_{33}$, and $d_{33}$ is a good starting point to find other piezoelectric alloys with similar or greater increases at lower computational cost than calculating all of the tensor coefficients of the three properties. 


\subsection{Modeling diffusion}

There are many ways to model atom diffusion, each with its own pros and cons. Of these, quantum molecular dynamics (QMD) has potential to be the most accurate one. The basic approach of this method is to calculate the forces between the atoms in a system with quantum mechanical accuracy, then evolve the system in time using these forces and updating their positions. The down side to this approach is the huge computational resources that are required to maintain this kind of accuracy. This limits the system to $\sim 100$ atoms and the simulation time to a couple of ns. However, the frequency of most diffusion events at low temperatures (compared to the melting temperature of nitrides ${ }^{4}$ ) is less than this simulation time, which makes it difficult to capture the relevant events.

With classical molecular dynamics (CMD), it is possible to simulate much larger systems ( 1000 atoms) for longer periods of time than QMD (a few $\mu \mathrm{s})$. Of course, the accuracy of this method relies on the precision of the force field parametrization, and increasing the accuracy will reduce the simulation time.

However, both QMD and CMD are impractical when the qualitative effects of alloying is of interest. Finding the correct parameters for each CMD force field or running enough QMD runs to find specific transitions require massive computational resources. A much more effective approach is to use DFT and focus on how alloying effects the potential energy barrier between transitions. Although DFT is a static approach which does not include any dynamics, the dominating parameter for diffusion at low temperatures is the potential energy barrier between sites. Moreover, one can argue that the effect of alloying on the vibrational freedoms is much less than the effect on the transition barrier when considering dilute cases (see Paper.III). The knowledge obtained by DFT can also be taken one step further to simulate growth by using the DFT parameters together with kinetic Monte Carlo (KMC) algorithms.

Regardless of the approach used to study diffusion, it is important to understand the relations between the relevant parameters affecting diffusion. The primary relations are described by Fick's two laws, which are described in the following sections.

\subsubsection{Fick's first law}

Consider a surface as shown in Fig. 2.4 (a). For a dilute concentration of surface adatoms, where no attempt will be blocked by occupied cites, the adatom movement to the right by jumping to unoccupied lattice sites with the flow $\vec{J}_{x}$ given by

$$
\overrightarrow{J_{x}}=\Gamma_{x} n_{1},
$$

where $\Gamma_{x}$ is the number of successful jumps in the $x$-direction per second, $n_{1}$ is the number of adatoms per unit length in line 1 . In the opposite direction from line 2 , where there are $n_{2}$ occupied sites along line 2 , the flow of adatoms is given by

\footnotetext{
${ }^{4}$ Melting temperature of TiN is $\sim 3200 \mathrm{~K}$.
} 


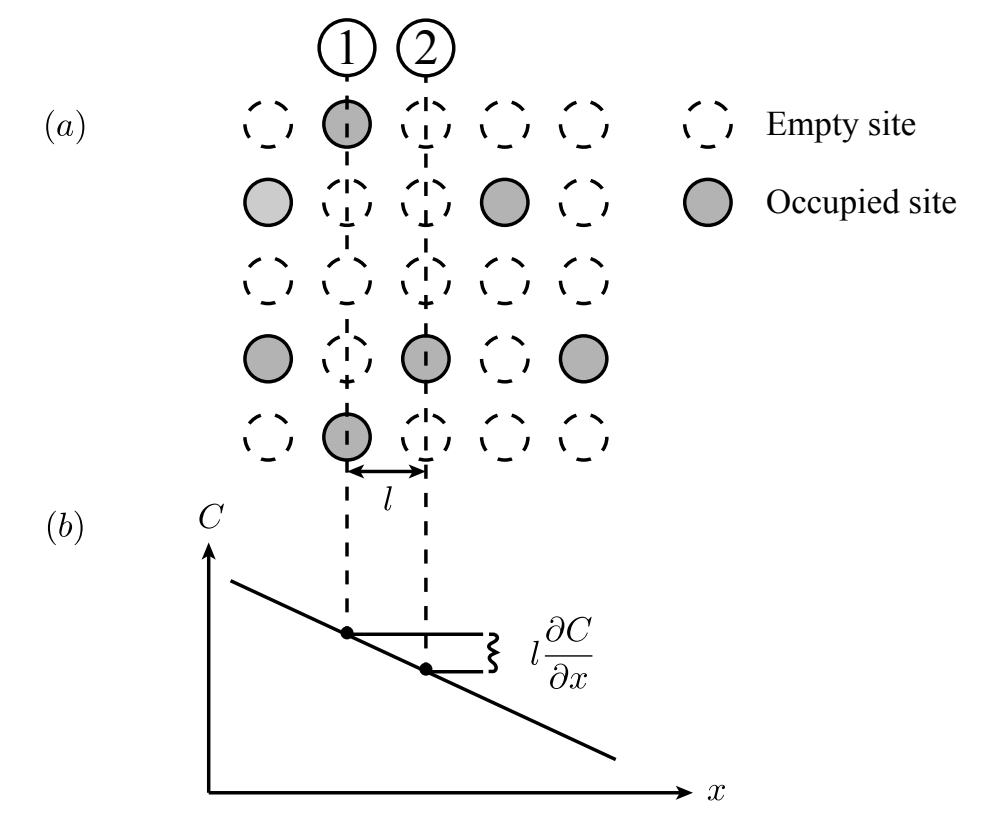

Figure 2.4. (a) A simple example of adatoms and empty sites on a cubic surface. (b) The concentration profile in the $x$-direction. Adapted from Ref.[79].

$$
\overleftarrow{J}_{x}=\Gamma_{x} n_{2}
$$

The difference between these two flows give us the resulting flow in the $\mathrm{x}$ direction $J_{x}$ as

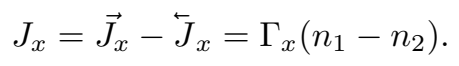

With $l$ as the separation between the lines, the concentration of adatoms in one line can be written as $C_{1}=n_{1} / l$ and $C_{2}=n 2 / l$, so that $\left(n_{1}-n_{2}\right)=l\left(C_{1}-C_{2}\right)$. The change in concentration shown in Fig. 2.4 (b) can be written as $\left(C_{1}-C_{2}\right)=$ $-l(\partial C / \partial x)$ using Taylor expansion, thus

$$
J_{x}=-\left(l^{2} \Gamma_{x}\right) \frac{\partial C}{\partial x} .
$$

Here we define the diffusivity as

$$
D=l^{2} \Gamma_{x}
$$

so that we end up with

$$
J=-D \frac{\partial C}{\partial x}
$$




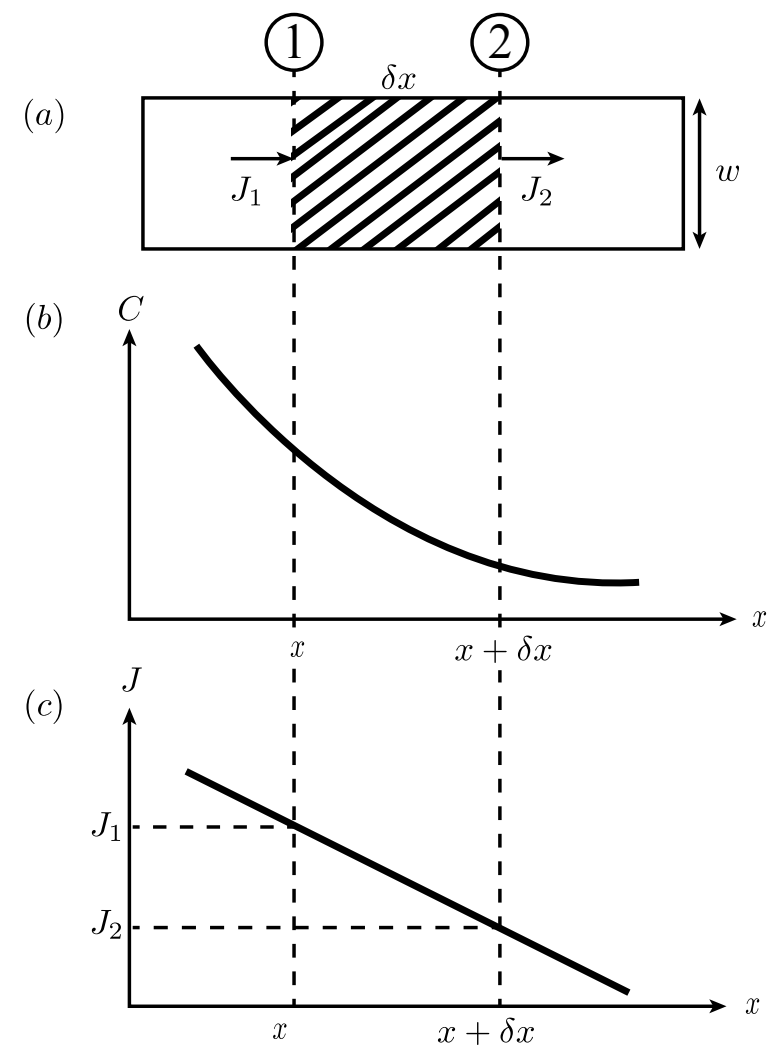

Figure 2.5. Derivation of Fick's second law. (a) A narrow band on the surface with width $w$ which adatoms enter with the flux $J_{1}$ and leaves with the flux $J_{2}$. (b) Concentration profile with respect to distance along the $x$-direction. (c) Corresponding adatom flows. Adapted from Ref. [79].

known as Fick's first law, suggested by Adolf Fick in 1855.[88, 89] Although the first law is enough for a steady-state situations, Fick's second law is needed to describe diffusion when the concentration is time dependent.

\subsubsection{Fick's second law}

For non steady-state systems, i.e. most practical systems, the concentration varies both with respect to time and distance. In order to calculate how the concentration at any point varies with time, we consider a narrow band on the surface with width $w$ and thickness $\delta x$, see Fig. 2.5 (a). The number of adatoms which diffuse into this band is given by

$$
\delta n_{1}=J_{1} w \delta t,
$$


and the number of adatoms diffusing out of the band is given by

$$
\delta n_{2}=J_{2} w \delta t
$$

The concentration of atoms within the band will change according to

$$
\delta C=\frac{\left(J_{1}-J_{2}\right) w \delta t}{w \delta x}
$$

And because $\delta x$ is small

$$
J_{2}=J_{1}+\frac{\partial J}{\partial x} \delta x
$$

In the limit where $\delta t \rightarrow 0$, Eqs.(2.66) and (2.67) give

$$
\frac{\partial C}{\partial t}=-\frac{\partial J}{\partial x}
$$

By substituting Fick's first law, Eq.(2.63),

$$
\frac{\partial C}{\partial t}=\frac{\partial}{\partial x}\left(D \frac{\partial C}{\partial x}\right)
$$

which simplifies to

$$
\frac{\partial C}{\partial t}=D \frac{\partial^{2} C}{\partial x^{2}}
$$

if $D$ is independent of $x$ and $C$.

\subsubsection{Fick's laws in more dimensions}

So far, the diffusion has been restricted to one dimension on a surface. However, expanding Fick's laws to cover more dimensions is a simple task. Using the nabla operator, Fick's laws can be written as

$$
J=-D \cdot \nabla C
$$

and

$$
\frac{\partial C}{\partial t}=D \cdot \nabla^{2} C
$$

where $C=C(x, y, z, t)$, and $D$ is a tensor. Here, it is pointed out that the $D$ can vary depending on the direction, although in a cubic lattice it is uniform in all directions.[90] 


\subsubsection{Temperature dependence}

The number of successful jumps any given atom can perform is dependent on temperature according to

$$
\Gamma=\nu \exp \left(-\frac{\Delta G_{m}}{k_{B} T}\right),
$$

where $\nu$ is the time independent vibration of the atoms, and $\Delta G$ is the free energy activation barrier which needs to be overcome in order for an adatom to migrate between jump sites separated by the distance $l$ [79]. Eq.(2.62) becomes

$$
D=l^{2} \nu \exp \left(-\frac{\Delta G}{k_{B} T}\right) .
$$

Substituting $\Delta G=\Delta H-T \Delta S$ gives

$$
D=l^{2} \exp \left(\frac{\Delta S}{k_{B}}\right) \exp \left(-\frac{\Delta H}{k_{B} T}\right) .
$$

The diffusivity $D$ can now be divided into one temperature dependent exponential factor and one temperature independent prefactor $D_{0}$, so that

$$
D=D_{0} \exp \left(-\frac{\Delta H}{k_{B} T}\right)
$$

and

$$
D_{0}=l^{2} \nu \exp \left(\frac{\Delta S}{k_{B}}\right)=l^{2} \nu_{0}
$$

where $\nu_{0}$ is the attempt frequency.

Assuming low pressures $\Delta H \approx \Delta E[90]$. The diffusivity is dependent on the diffusion activation barrier $\Delta E$ according to

$$
D=D_{0} \exp \left(-\frac{\Delta E}{k_{B} T}\right)
$$

Which factor, $D_{0}$ or $\Delta E$ that will be the dominating one is determined by the temperature. The diffusion activation barrier will be dominating the diffusivity at low temperatures, and at least in principle at high temperatures $D \rightarrow D_{0}$.

\subsubsection{Calculating the diffusion activation barrier $\Delta E$}

The barrier is determined by the difference in the adatom adsorption energy $E_{a d}$ between a binding site and a saddle point, see Fig. 2.6. The adsorption energy $E_{a d}$ at the coordinate $(x, y)$ is calculated as

$$
E_{a d}(x, y)=E_{\text {config }}(x, y)-\left(E_{\text {slab }}+E_{\text {atom }}^{\text {vacuum }}\right),
$$

the difference between a slab with an adatom and a slab where the adatom is in vacuum. 


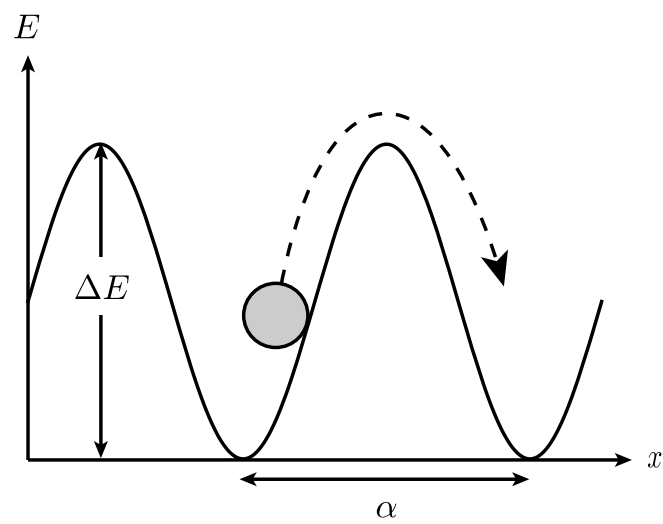

Figure 2.6. The diffusion activation barrier $\Delta E$ is the difference in adsorption energy between a binding site and a saddle point.

Calculating the adsorption energy is a simple task. The difficulty in obtaining the diffusion activation barrier is finding the binding sites and saddle points. Although this is easy for a simple cubic cell with only one type of atomic species, the problem becomes many times more complex for alloys with impurities. Two types of methods have been used in this work to find the binding sites and saddle points; by probing the entire surface to find the entire adsorption energy landscape, and using the nudged elastic band (NEB) method.

\section{Grid method}

In the grid method, the adsorption energy landscape is calculated by probing the adsorption energy of an adatom at different positions on the surface by fixing the in-plane relaxation allowing only relaxation perpendicular to the surface, see Fig. 2.7 (a).

The accuracy of the adsorption energy landscape is limited by the grid mesh. The grid needs to be fine enough to capture the entire topology of the adsorption energy landscape. This requires many calculations to reach a high accuracy. However, the number of calculations needed can be reduced using the symmetry of the surfaces. Also, since the calculations are independent of each other they don't need to be run at the same time.

Calculating the adsorption energy landscape gives an excellent overview of the diffusion landscape, however, it is not as accurate at calculate barrier heights as the NEB method.

\section{Nudged elastic band method}

The nudged elastic band (NEB) method [91-93] finds the lowest energy path between two binding sites by simultaneously calculating the potential energy at positions. Initially, a path between two minima is set up with estimated adatom 

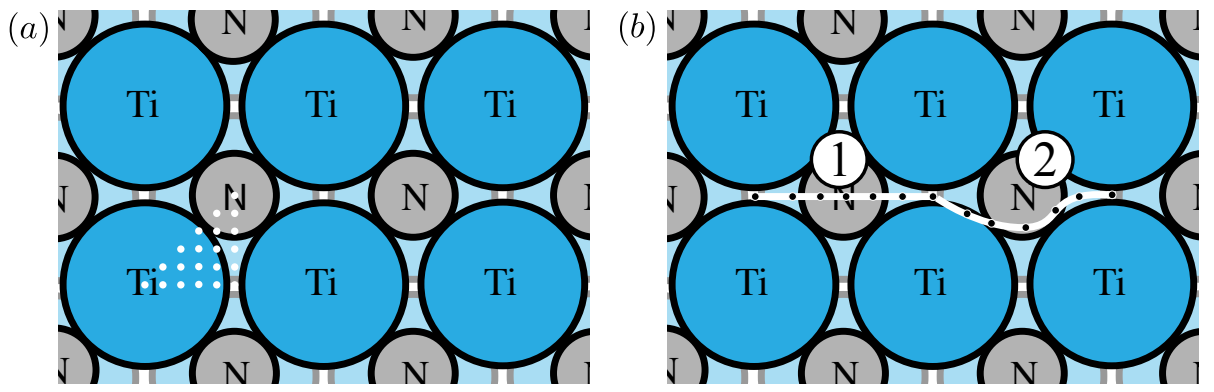

Figure 2.7. (a) Setup for calculating the adsorbtion energetics by probing the surface in a grid, where the adatom is fixed in-plane position. (b) Illustration of the relaxing effect using the NEB method. The method allows for a linear path 1 to relax into a lowest energy path 2 .

positions, through for example linear interpolation, see Fig. 2.7 (b) 1. The adatom positions are then simultaneously relaxed in the $x, y$, and $z$ directions to find the minimum energy path (Fig. 2.7). The relaxations are restricted by a spring force, so that the images are evenly spaced, while making sure the saddle points are included.

This relaxation method used within the NEB method gives a more accurate minimum energy path with fewer calculations than the previous method. However, to effectively use the method, all minima need to be known in order to set up the path. The binding sites depend strongly on the adatom species, so in most cases it is helpful to first get an overview using the grid approach with a rough grid.

\subsubsection{Approximating the diffusion prefactor}

Estimating the diffusion prefactor $D_{0}$ is difficult both experimentally and theoretically. However, for diffusion of isolated individual adatoms on a surface the fluctuations in the prefactor are generally small and differ little between systems. Still, there are ways to approximate it. Vineyard [40] showed that the prefactor can generally be written as

$$
\nu=\frac{\prod_{j=1}^{n} \nu_{j}}{\prod_{i=1}^{n-1} \nu_{i}},
$$

the ratio of all $n$ vibrations in the system when the adatom is at a binding site and all $n-1$ vibrations when it is at the saddle point. Calculating all of these vibrations is very costly. However, with a simple approximation, that all vibrations other than the adatom vibration in the direction of the jump path are constant, the number of vibrations that are needed are reduced to just one, the vibration frequency when the adatom is at a binding site $\nu_{0}$. Although this approximation 

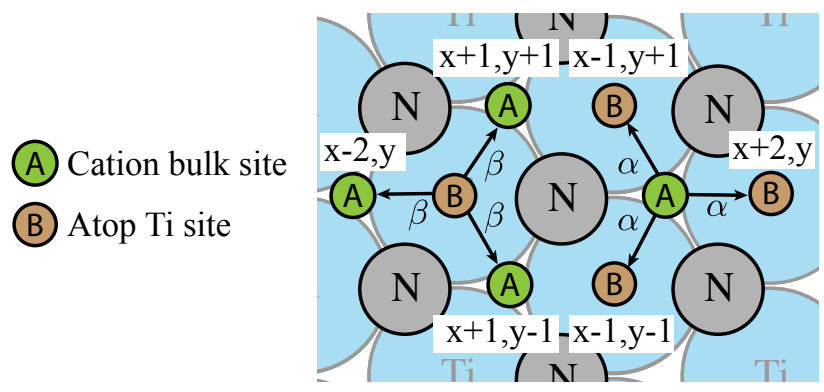

Figure 2.8. Binding site arrangement on a $\operatorname{TiN}(111): \mathrm{N}$ surface where the jump rate from $\mathrm{A}$ to $\mathrm{B}$ is $\alpha$ and the jump rate from $\mathrm{B}$ to $\mathrm{A}$ is $\beta$.

does not hold for diffusion mechanisms which requires movement of multiple atoms simultaneously, it is accurate enough to give a first order description of adatom surface diffusion.

$\nu_{0}$ can be approximated in a classical sense by treating the atom at a binding site as a harmonic oscillator using

$$
\nu_{0}=\frac{1}{2 \pi} \sqrt{\frac{\partial^{2} E / \partial x^{2}}{m}},
$$

where $\partial^{2} E / \partial x^{2}$ is the second derivative of potential energy at the binding site and $m$ is the mass of the adatom. Although this is a crude approximation, errors in the diffusivity caused by this approximation is shadowed by the general transition state theory approximations.

\subsubsection{Calculating the diffusivity on surfaces when there is more than one type of binding site}

Calculating the surface diffusivity for systems with only one type of binding site and one atomic species (e.g., a simple cubic (001) surface) is a relatively simple task, which is probably why it is a common example in the available literature on diffusion. However, when considering alloy systems the problem can become quite complex. Some adatom species may have more than one binding site which makes it more difficult to compare the diffusivity of the two species. This added complexity can be seen on the N-terminated $\operatorname{TiN}(111)$ surface, Fig. 2.8. An expression for the diffusivity can be found by following the method proposed by Wrigley, Twigg, and Ehrlich [94].

According to Wrigley, Twigg, and Ehrlich [94], the probability that an adatom is at an A site with the coordinates $(x, y)$ is $p_{x, y}^{A}$, and the probability that it is a $\mathrm{B}$ site with the coordinates $(x, y)$ is $p_{x, y}^{B}$. Because the binding sites are connected as in Fig. 2.8, considering only nearest neighbor jumps with the jump rates are $\Gamma_{\alpha}$ and $\Gamma_{\beta}$ from an $\mathrm{A}$ site respectively $\mathrm{B}$ site, the time rate of change of the probabilities are described by the Kolmogoroff equations [95] 


$$
\frac{d p_{x, y}^{A}}{d t}=-3 \Gamma_{\alpha} p_{x, y}^{A}+\Gamma_{\beta}\left(p_{x+2, y}^{B}+p_{x-1, y+1}^{B}+p_{x-1, y-1}^{B}\right)
$$

and

$$
\frac{d p_{x, y}^{B}}{d t}=-3 \Gamma_{\beta} p_{x, y}^{A}+\Gamma_{\alpha}\left(p_{x-2, y}^{B}+p_{x+1, y+1}^{B}+p_{x+1, y-1}^{B}\right) .
$$

The probability generating functions for the sites are defined as

$$
G^{A}\left(t, z_{1}, z_{2}\right)=\sum_{x} \sum_{y} z_{1}^{x} z_{2}^{y} p_{x, y}^{A}
$$

and

$$
G^{B}\left(t, z_{1}, z_{2}\right)=\sum_{x} \sum_{y} z_{1}^{x} z_{2}^{y} p_{x, y}^{B},
$$

where the sums are taken over the $\mathrm{A}$ and $\mathrm{B}$ sites respectively, and $z_{1}$ and $z_{2}$ are dummy variables in the range $0 \leq z \leq 1$. The generating functions are related to the total generating function by

$$
G\left(t, z_{1}, z_{2}\right)=G^{A}\left(t, z_{1}, z_{2}\right)+G^{B}\left(t, z_{1}, z_{2}\right) .
$$

The time derivative of the A site generating function is

$$
\begin{aligned}
\dot{G}^{A}\left(t, z_{1}, z_{2}\right)= & \sum_{x} \sum_{y} z_{1}^{x} z_{2}^{y} \frac{d p_{x, y}^{A}}{d t}= \\
& -\Gamma_{\alpha} \sum_{x} \sum_{y} p_{x, y}^{A}+\Gamma_{\beta}\left(\sum_{x} \sum_{y} z_{1}^{x} z_{2}^{y} p_{x+2, y}^{B}\right. \\
& \left.+\sum_{x} \sum_{y} z_{1}^{x} z_{2}^{y} p_{x-1, y+1}^{B}+\sum_{x} \sum_{y} z_{1}^{x} z_{2}^{y} p_{x-1, y-1}^{B}\right),
\end{aligned}
$$

which can be more conveniently written as

$$
\begin{aligned}
\dot{G}^{A}\left(t, z_{1}, z_{2}\right)= & -3 \Gamma_{\alpha} \sum_{x} \sum_{y} p_{x, y}^{A} \\
+\Gamma_{\beta}( & \frac{1}{z_{1}^{2}} \sum_{x} \sum_{y} z_{1}^{x+2} z_{2}^{y} p_{x+2, y}^{B} \\
& +\frac{z_{1}}{z_{2}} \sum_{x} \sum_{y} z_{1}^{x-1} z_{2}^{y+1} p_{x-1, y+1}^{B} \\
& \left.+z_{1} z_{2} \sum_{x} \sum_{y} z_{1}^{x-1} z_{2}^{y-1} p_{x-1, y-1}^{B}\right) .
\end{aligned}
$$

A shorter way to represent the equation is 


$$
\begin{aligned}
\dot{G}^{A}\left(t, z_{1}, z_{2}\right)= & -3 \Gamma_{\alpha} G^{A}\left(t, z_{1}, z_{2}\right) \\
& +\Gamma_{\beta}\left(\frac{1}{z_{2}^{2}}+\frac{z_{1}}{z_{2}}+z_{1} z_{2}\right) G^{B}\left(t, z_{1}, z_{2}\right) .
\end{aligned}
$$

In the same way

$$
\begin{aligned}
\dot{G}^{B}\left(t, z_{1}, z_{2}\right)= & -3 \Gamma_{\beta} G^{B}\left(t, z_{1}, z_{2}\right) \\
& +\Gamma_{\alpha}\left(z_{1}^{2}+\frac{1}{z_{1} z_{2}}+\frac{z_{2}}{z_{1}}\right) G^{A}\left(t, z_{1}, z_{2}\right) .
\end{aligned}
$$

with

$$
\begin{aligned}
& u=\frac{1}{3}\left(\frac{1}{z_{2}^{2}}+\frac{z_{1}}{z_{2}}+z_{1} z_{2}\right), \\
& v=\frac{1}{3}\left(z_{1}^{2}+\frac{1}{z_{1} z_{2}}+\frac{z_{2}}{z_{1}}\right) .
\end{aligned}
$$

Eq. (2.89) and (2.90) can be written as

$$
\begin{aligned}
& \dot{G}^{A}\left(t, z_{1}, z_{2}\right)=-3 \Gamma_{\alpha} G^{A}\left(t, z_{1}, z_{2}\right)+3 \Gamma_{\beta} u G^{B}\left(t, z_{1}, z_{2}\right), \\
& \dot{G}^{B}\left(t, z_{1}, z_{2}\right)=-3 \Gamma_{\beta} G^{B}\left(t, z_{1}, z_{2}\right)+3 \Gamma_{\alpha} v G^{A}\left(t, z_{1}, z_{2}\right) .
\end{aligned}
$$

If the adatom starts at the $(x, y)=(0,0)$ position then $G^{A}\left(0, z_{1}, z_{2}\right)=z_{1} z_{2}$ and $G^{B}\left(0, z_{1}, z_{2}\right)=0 .{ }^{5}$ Thus, the Laplace transform of the left hand sides of Eq.(2.93) and $(2.94)$ are

$$
\begin{aligned}
\mathcal{L}\left(\dot{G}^{A}\left(t, z_{1}, z_{2}\right)\right)= & s \mathcal{L}\left(G^{A}\left(t, z_{1}, z_{2}\right)\right)+G^{A}\left(0, z_{1}, z_{2}\right)= \\
& s \mathcal{L}\left(G^{A}\left(t, z_{1}, z_{2}\right)\right)+z_{1} z_{2}, \\
\mathcal{L}\left(\dot{G}^{B}\left(t, z_{1}, z_{2}\right)\right)= & s \mathcal{L}\left(G^{B}\left(t, z_{1}, z_{2}\right)\right)+G^{B}\left(0, z_{1}, z_{2}\right)= \\
& s \mathcal{L}\left(G^{B}\left(t, z_{1}, z_{2}\right)\right),
\end{aligned}
$$

and the right hand sides are

$$
-3 \Gamma_{\alpha} \mathcal{L}\left(G^{A}\left(t, z_{1}, z_{2}\right)\right)+3 \Gamma_{\beta} u \mathcal{L}\left(G^{B}\left(t, z_{1}, z_{2}\right)\right)
$$

\footnotetext{
${ }^{5}$ It is also possible to choose the B-site as the starting position. Then $G^{A}\left(0, z_{1}, z_{2}\right)=0$ and $G^{B}\left(0, z_{1}, z_{2}\right)=z_{1}^{2}$.
} 


$$
-3 \Gamma_{\beta} \mathcal{L}\left(G^{B}\left(t, z_{1}, z_{2}\right)\right)+3 \Gamma_{\alpha} v \mathcal{L} .\left(G^{A}\left(t, z_{1}, z_{2}\right)\right) .
$$

Solving the equation system gives

$$
\begin{aligned}
& \mathcal{L}\left(G^{A}\left(t, z_{1}, z_{2}\right)\right)=\frac{\left(s+3 \Gamma_{\beta}\right) z_{1} z_{2}}{(s+q)(s+r)}, \\
& \mathcal{L}\left(G^{B}\left(t, z_{1}, z_{2}\right)\right)=\frac{3 v \Gamma_{\alpha} z_{1} z_{2}}{(s+q)(s+r)},
\end{aligned}
$$

where

$$
\begin{gathered}
q \equiv \frac{3\left(\Gamma_{\alpha}+\Gamma_{\beta}\right)}{2}\left(1+\sqrt{1-\frac{4 \Gamma_{\alpha} \Gamma_{\beta}(1-u v)}{\left(\Gamma_{\alpha}+\Gamma_{\beta}\right)^{2}}}\right), \\
r \equiv \frac{3\left(\Gamma_{\alpha}+\Gamma_{\beta}\right)}{2}\left(1-\sqrt{1-\frac{4 \Gamma_{\alpha} \Gamma_{\beta}(1-u v)}{\left(\Gamma_{\alpha}+\Gamma_{\beta}\right)^{2}}}\right) .
\end{gathered}
$$

The inverse Laplace transforms of Eq.2.99 and 2.100 will then give the generating functions

$$
G^{A}\left(t, z_{1}, z_{2}\right)=\frac{z_{1} z_{2}}{q-r}\left(\left(q-3 \Gamma_{\beta}\right) e^{-q t}-\left(r-3 \Gamma_{\beta}\right) e^{-r t}\right)
$$

and

$$
G^{B}\left(t, z_{1}, z_{2}\right)=\frac{3 v \Gamma_{\alpha} z_{1} z_{2}}{q-r}\left(e^{-r t}-e^{-q t}\right) .
$$

The probability $P^{A}$ that an adatom is at an A site is simply $G^{A}(t, 1,1)$, since

$$
\begin{array}{r}
P^{A}=\sum_{x} \sum_{y} p_{x, y}^{A}=G^{A}(t, 1,1) \\
=\frac{\Gamma_{\alpha} e^{-3\left(\Gamma_{\alpha}+\Gamma_{\beta}\right) t}+\Gamma_{\beta}}{\Gamma_{\alpha}+\Gamma_{\beta}} .
\end{array}
$$

The probability $P^{B}$ is likewise

$$
\begin{array}{r}
P^{B}=\sum_{x} \sum_{y} p_{x, y}^{B}=G^{B}(t, 1,1) \\
=\frac{\Gamma_{\alpha} e^{-3\left(\Gamma_{\alpha}+\Gamma_{\beta}\right) t}-\Gamma_{\alpha}}{\Gamma_{\alpha}+\Gamma_{\beta}} .
\end{array}
$$

From the generating function it is now possible to access the moments in the $x$-direction $\left\langle x^{n}\right\rangle$ through the relation 


$$
\left\langle x^{n}\right\rangle=\sum_{x} x^{n} p_{x}=\left[\left(z_{1} \frac{\partial}{\partial z_{1}}\right)^{n} G\left(t, z_{1}, 1\right)\right]_{z_{1}=1}
$$

where moments are in the units $a / \sqrt{6}$, where $a$ is the lattice constant ${ }^{6}$. The average displacement $\langle x\rangle$ is

$$
\langle x\rangle=1
$$

and the mean-square value $\left\langle x^{2}\right\rangle$ is

$$
\left\langle x^{2}\right\rangle=\frac{2 \Gamma_{\alpha}\left(\Gamma_{\beta}-\Gamma_{\alpha}\right) e^{-3\left(\Gamma_{\alpha}+\Gamma_{\beta}\right) t}+\Gamma_{\beta}^{2}+12 \Gamma_{\alpha} \Gamma_{\beta}^{2} t+3 \Gamma_{\alpha}^{2}\left(1+4 \Gamma_{\beta} t\right)}{\left(\Gamma_{\alpha}+\Gamma_{\beta}\right)^{2}}
$$

The fluctuation $\left\langle\Delta x^{2}\right\rangle$ in $x$ is

$$
\left\langle\Delta x^{2}\right\rangle=\left\langle x^{2}\right\rangle-\langle x\rangle^{2}=\frac{2 \Gamma_{\alpha}\left(\Gamma_{\alpha}-\Gamma_{\beta}\right)\left(1-e^{-3\left(\Gamma_{\alpha}+\Gamma_{\beta}\right) t}\right)}{\left(\Gamma_{\alpha}+\Gamma_{\beta}\right)^{2}}+\frac{12 \Gamma_{\alpha} \Gamma_{\beta} t}{\Gamma_{\alpha}+\Gamma_{\beta}} .
$$

The first term in Eq.(2.110) accounts for the transient behavior at the beginning of diffusion where the starting site is important. For long diffusion times, the equation can be reduced to

$$
\left\langle\Delta x^{2}\right\rangle=12 \frac{\Gamma_{\alpha} \Gamma_{\beta} t}{\Gamma_{\alpha}+\Gamma_{\beta}}
$$

where $\mathrm{x}$ is still in units of $a / \sqrt{6}$, converted to $\mathrm{m}^{2} / \mathrm{s}$ the equation becomes

$$
\left\langle\Delta a^{2}\right\rangle=2 a^{2} \frac{\Gamma_{\alpha} \Gamma_{\beta} t}{\Gamma_{\alpha}+\Gamma_{\beta}}
$$

The diffusivity $\mathrm{D}$ is derived from the fluctuation $\left\langle\Delta a^{2}\right\rangle$ through the Einstein relation

$$
\left\langle\Delta a^{2}\right\rangle=2 D t
$$

which means that the surface diffusivity is

$$
D=a^{2} \frac{\Gamma_{\alpha} \Gamma_{\beta}}{\Gamma_{\alpha}+\Gamma_{\beta}} .
$$

Although the SI unit of $D$ is $\mathrm{m}^{2} / \mathrm{s}$, a commonly used unit is $\mathrm{cm}^{2} / \mathrm{s}$.

\footnotetext{
${ }^{6}$ Moments in the $y$-direction will in this symmetry be in the unit $a /(2 \sqrt{2})$
} 
[1] S. P. McPherron et al. "Evidence for stone-tool-assisted consumption of animal tissues before 3.39 million years ago at Dikika, Ethiopia." In: Nature 466.7308 (Aug. 2010), pp. 857-60.

[2] R. E. Hummel. Understanding Materials Science: History, Properties, Applications, Second Edition. 2nd. SpringerLink : Bücher. Springer, 2004.

[3] M. Ohring. Materials Science of Thin Films. Elsevier Science, 2001.

[4] G. Antczak and G. Ehrlich. Surface Diffusion: Metals, Metal Atoms, and Clusters. First. Cambridge: Cambridge University Press, 2010, p. 784.

[5] D. Gall et al. "Pathways of atomistic processes on TiN (001) and (111) surfaces during film growth: an ab initio study". In: J. Appl. Phys. 93.11 (2003), pp. 9086-9094.

[6] Z. L. Wang. "Towards Self-Powered Nanosystems: From Nanogenerators to Nanopiezotronics". In: Adv. Funct. Mater. 18.22 (Nov. 2008), pp. 3553-3567.

[7] A. Hajati and S.-G. Kim. "Ultra-wide bandwidth piezoelectric energy harvesting". In: Appl. Phys. Lett. 99.8 (Aug. 2011), p. 083105.

[8] K. Hashimoto et al. "High-performance surface acoustic wave resonators in the 1 to $3 \mathrm{GHz}$ range using a ScAlN/6H-SiC structure." In: IEEE Trans. Ultrason. Ferroelectr. Freq. Control 60.3 (Mar. 2013), pp. 637-42.

[9] K. Umeda et al. "Piezoelectric properties of ScAlN thin films for piezo-MEMS devices". In: 2013 IEEE 26th Int. Conf. Micro Electro Mech. Syst. IEEE, Jan. 2013, pp. 733-736.

[10] M. Suzuki, T. Yanagitani, and H. Odagawa. "Polarity-inverted ScAlN film growth by ion beam irradiation and application to overtone acoustic wave $(000 \overline{1}) /\left(\begin{array}{lll}0 & 0 & 1\end{array}\right)$ film resonators". In: Appl. Phys. Lett. 104.17 (Apr. 2014), p. 172905. 
[11] M. Akiyama et al. "Enhancement of Piezoelectric Response in Scandium Aluminum Nitride Alloy Thin Films Prepared by Dual Reactive Cosputtering". In: Adv. Mater. 21.5 (2008), pp. 593-596.

[12] Y. Saito et al. "Lead-free piezoceramics". In: Nature 432 (Nov. 2004), pp. 25 .

[13] N. D. Patel and P. S. Nicholson. "High frequency, high temperature ultrasonic transducers". In: NDT Int. 23.5 (Oct. 1990), pp. 262-266.

[14] F. Tasnádi et al. "Origin of the Anomalous Piezoelectric Response in Wurtzite $\mathrm{Sc}_{x} \mathrm{Al}_{1-x} \mathrm{~N}$ Alloys". In: Phys. Rev. Lett. 104.13 (Apr. 2010), p. 137601.

[15] S. Zhang et al. "Elastic constants and critical thicknesses of ScGaN and ScAlN". In: J. Appl. Phys. 114.24 (Dec. 2013), p. 243516.

[16] T. Yanagitani and M. Suzuki. "Enhanced piezoelectricity in YbGaN films near phase boundary". In: Appl. Phys. Lett. 104.8 (Feb. 2014), p. 082911.

[17] M. A. Moram and S. Zhang. "ScGaN and ScAlN: emerging nitride materials". In: J. Mater. Chem. A 2.17 (Apr. 2014), pp. 6042-6050.

[18] T. Leyendecker et al. "The development of the PVD coating TiAlN as a commercial coating for cutting tools". In: Surf. Coatings Technol. 48.2 (1991), pp. $175-178$.

[19] B. Subramanian et al. "A comparative study of titanium nitride (TiN), titanium oxy nitride (TiON) and titanium aluminum nitride (TiAlN), as surface coatings for bio implants". In: Surf. Coatings Technol. 205.21-22 (2011), pp. 5014-5020.

[20] G. Beensh-Marchwicka, L. Kròl-Stpniewska, and W. Posadowski. "Structure of thin films prepared by the cosputtering of titanium and aluminium or titanium and silicon". In: Thin Solid Films 82.4 (1981), pp. 313-320.

[21] H. A. Jehn et al. "Morphology and properties of sputtered (Ti,Al)N layers on high speed steel substrates as a function of deposition temperature and sputtering atmosphere". In: J. Vac. Sci. Technol. A Vacuum, Surfaces, Film. 4.6 (Nov. 1986), p. 2701.

[22] O. Knotek, M. Böhmer, and T. Leyendecker. "On structure and properties of sputtered Ti and Al based hard compound films". In: J. Vac. Sci. Technol. A Vacuum, Surfaces, Film. 4.6 (Nov. 1986), p. 2695.

[23] O. Knotek, W. D. Münz, and T. Leyendecker. "Industrial deposition of binary, ternary, and quaternary nitrides of titanium, zirconium, and aluminum". In: J. Vac. Sci. Technol. A Vacuum, Surfaces, Film. 5.4 (July 1987), p. 2173.

[24] P. C. Jindal et al. "Performance of PVD TiN, TiCN, and TiAlN coated cemented carbide tools in turning". In: Int. J. Refract. Met. Hard Mater. 17.1 (1999), pp. 163-170.

[25] A. Hörling et al. "Mechanical properties and machining performance of $\mathrm{Ti}(1 \mathrm{x}) \mathrm{Al}(\mathrm{x}) \mathrm{N}$-coated cutting tools". In: Surf. Coatings Technol. 191.2-3 (Feb. 2005), pp. 384-392. 
[26] W.-D. Münz. "Titanium aluminum nitride films: A new alternative to TiN coatings". In: J. Vac. Sci. Technol. A Vacuum, Surfaces, Film. 4.6 (Nov. 1986), p. 2717.

[27] D. McIntyre et al. "Oxidation of metastable single-phase polycrystalline $\mathrm{Ti}(0.5) \mathrm{Al}(0.5) \mathrm{N}$ films: Kinetics and mechanisms". In: J. Appl. Phys. 67.3 (1990), p. 1542.

[28] Y. C. Chim et al. "Oxidation resistance of TiN, CrN, TiAlN and CrAlN coatings deposited by lateral rotating cathode arc". In: Thin Solid Films 517.17 (2009), pp. 4845-4849.

[29] D. Music and J. M. Schneider. "Ab initio study of $\mathrm{Ti}_{0.5} \mathrm{Al}_{0.5} \mathrm{~N}(001)$ residual and environmental gas interactions". In: New J. Phys. 15.7 (July 2013), p. 073004.

[30] P. H. Mayrhofer et al. "Self-organized nanostructures in the TiAlN system". In: Appl. Phys. Lett. 83 (2003), p. 2049.

[31] A. E. Santana et al. "Thermal treatment effects on microstructure and mechanical properties of TiAlN thin films". In: Tribol. Lett. 17.4 (Nov. 2004), pp. 689-696.

[32] R. Rachbauer et al. "Decomposition pathways in age hardening of Ti-Al-N films". In: J. Appl. Phys. 110 (2011), p. 023515.

[33] L. J. S. Johnson et al. "Spinodal decomposition of $\mathrm{Ti}_{0.33} \mathrm{Al}_{0.67} \mathrm{~N}$ thin films studied by atom probe tomography". In: Thin Solid Films 520.13 (2012), pp. $4362-4368$.

[34] A. Knutsson et al. "Microstructure evolution during the isostructural decomposition of TiAlN - A combined in-situ small angle x-ray scattering and phase field study". In: J. Appl. Phys. 113.21 (June 2013), p. 213518.

[35] A. Knutsson et al. "Surface directed spinodal decomposition at TiAlN/TiN interfaces". In: J. Appl. Phys. 113.11 (2013), p. 114305.

[36] B. Alling et al. "Mixing and decomposition thermodynamics of $\mathrm{c}-\mathrm{Ti}_{1-x} \mathrm{Al}_{x} \mathrm{~N}$ from first-principles calculations". In: Phys. Rev. B 75 (2007), p. 045123.

[37] B. Alling, A. Karimi, and I. A. Abrikosov. "Electronic origin of the isostructural decomposition in cubic $\mathrm{M}_{1-x} \mathrm{Al}_{x} \mathrm{~N}$ (M=Ti, Cr, Sc, Hf): A first-principles study". In: Surf. Coatings Technol. 203.5-7 (Dec. 2008), pp. 883-886.

[38] B. Alling et al. "Pressure enhancement of the isostructural cubic decomposition in $\mathrm{Ti}_{1-x} \mathrm{Al}_{x} \mathrm{~N}$ ". In: Appl. Phys. Lett. 95.18 (2009), p. 181906.

[39] H. Eyring. "The Activated Complex in Chemical Reactions". In: J. Chem. Phys. 3.2 (Feb. 1935), p. 107.

[40] G. H. Vineyard. "Frequency factors and isotope effects in solid state rate processes". In: J. Phys. Chem. Solids 3.1-2 (1957), pp. 121-127.

[41] A. Bogicevic, J. Strömquist, and B. I. Lundqvist. "Low-Symmetry Diffusion Barriers in Homoepitaxial Growth of $\mathrm{Al}(111)$ ". In: Phys. Rev. Lett. 81.3 (1998), pp. 637-640. 
[42] M. Mantina et al. "First-Principles Calculation of Self-Diffusion Coefficients". In: Phys. Rev. Lett. 100.21 (May 2008), p. 215901.

[43] C. Ruberto, A. Vojvodic, and B. I. Lundqvist. "Nature of versatile chemisorption on TiC(111) and TiN(111) surfaces". In: Solid State Commun. 141.1 (2007), pp. 48-52.

[44] C. Ruberto and B. I. Lundqvist. "Nature of adsorption on $\mathrm{TiC}(111)$ investigated with density-functional calculations". In: Phys. Rev. B 75.23 (2007), p. 235438.

[45] C. V. Ciobanu, D. T. Tambe, and V. B. Shenoy. "First-principles calculations of step formation energies and step interactions on TiN(001)". In: Surf. Sci. 582.1-3 (2005), pp. 145-150.

[46] J. Graciani et al. "Interaction of oxygen with TiN(001):NO exchange and oxidation process". In: J. Chem. Phys. 126.24 (2007), pp. 244713-244718.

[47] S. Sanyal, U. V. Waghmare, and J. A. Ruud. "Adsorption of water on TiN (100), (110) and (111) surfaces: A first-principles study". In: Appl. Surf. Sci. 257.15 (2011), pp. 6462-6467.

[48] T. Akiyama, K. Nakamura, and T. Ito. "Ab initio-based study for adatom kinetics on $\mathrm{AlN}(0001)$ surfaces during metal-organic vapor-phase epitaxy growth". In: Appl. Phys. Lett. 100.25 (2012), p. 251601.

[49] M. Born and R. Oppenheimer. "Zur Quantentheorie der Molekeln". In: Ann. Phys. 389.20 (1927), pp. 457-484.

[50] F. Bloch. "Über die Quantenmechanik der Elektronen in Kristallgittern". In: Zeitschrift für Phys. 52.7-8 (July 1929), pp. 555-600.

[51] L. H. Thomas. "The calculation of atomic fields". In: Math. Proc. Cambridge Philos. Soc. 23.05 (Oct. 1927), p. 542.

[52] E. Fermi. "Un metodo statistico per la determinazione di alcune priorieta dellatome". In: Rend. Accad. Naz. Lincei 6.602-607 (1927), p. 32.

[53] P. Hohenberg and W. Kohn. "Inhomogeneous Electron Gas". In: Phys. Rev. 136 (1964), B864-B871.

[54] R. M. Martin. Electronic Structure: Basic Theory and Practical Methods. Cambridge University Press, 2004.

[55] W. Kohn and L. J. Sham. "Self-Consistent Equations Including Exchange and Correlation Effects". In: Phys. Rev. 140.4A (1965), A1133-A1138.

[56] W. Kohn. "Nobel Lecture: Electronic structure of matterwave functions and density functionals". In: Rev. Mod. Phys. 71.5 (Oct. 1999), pp. 1253-1266.

[57] U. von Barth and L. Hedin. "A local exchange-correlation potential for the spin polarized case. i". In: J. Phys. C Solid State Phys. 5.13 (July 1972), pp. 1629-1642.

[58] D. M. Ceperley and B. J. Alder. "Ground State of the Electron Gas by a Stochastic Method". In: Phys. Rev. Lett. 45.7 (1980). 
[59] S. H. Vosko, L. Wilk, and M. Nusair. "Accurate spin-dependent electron liquid correlation energies for local spin density calculations: a critical analysis". In: Can. J. Phys. 58.8 (Aug. 1980), pp. 1200-1211.

[60] J. P. Perdew and A. Zunger. "Self-interaction correction to density-functional approximations for many-electron systems". In: Phys. Rev. B 23.10 (1981), p. 5048 .

[61] A. D. Becke. "Density-functional exchange-energy approximation with correct asymptotic behavior". In: Phys. Rev. A 38.6 (1988), pp. 3098-3100.

[62] J. P. Perdew and Y. Wang. "Accurate and simple analytic representation of the electron-gas correlation energy". In: Phys. Rev. B 45.23 (1992), pp. 244249.

[63] J. P. Perdew, K. Burke, and M. Ernzerhof. "Generalized gradient approximation made simple". In: Phys. Rev. Lett. Phys. Rev. Lett. (USA) 77.18 (Oct. 1996), pp. 3865-3868.

[64] B. Hammer, L. Hansen, and J. Nørskov. "Improved adsorption energetics within density-functional theory using revised Perdew-Burke-Ernzerhof functionals". In: Phys. Rev. B 59.11 (Mar. 1999), pp. 7413-7421.

[65] J. P. Perdew, K. Burke, and M. Ernzerhof. "Erratum: Generalized Gradient Approximation Made Simple." In: Phys. Rev. Lett. 78 (Oct. 1997), p. 1396.

[66] VI Anisimov, Jan Zaanen, and OK Andersen. "Band theory and Mott insulators: Hubbard U instead of Stoner I". In: Phys. Rev. B 44.3 (1991).

[67] V. I. Anisimov, I. V. Solovyev, and M. A. Korotin. "Density-functional theory and NiO photoemission spectra". In: Phys. Rev. B 48.23 (1993).

[68] V. I. Anisimov, F. Aryasetiawan, and A. I. Lichtenstein. "First-principles calculations of the electronic structure and spectra of strongly correlated systems: the LDA + U method". In: J. Phys. Condens. Matter 9 (1997), p. 767 .

[69] C. Lee, W. Yang, and R. G. Parr. "Development of the Colic-Salvetti correlation-energy formula into a functional of the electron density". In: Phys. Rev. B 37.2 (1988).

[70] L. Schimka et al. "Accurate surface and adsorption energies from many-body perturbation theory". In: Nat. Mater. 9.9 (Sept. 2010), pp. 741-744.

[71] D. Vanderbilt. "Soft self-consistent pseudopotentials in a generalized eigenvalue formalism". In: Phys. Rev. B 41.11 (Dec. 1990), p. 7892.

[72] P. E. Blöchl. "Projector augmented-wave method". In: Phys. Rev. B 50.24 (Dec. 1994), pp. 17953-17979.

[73] G. Kresse and D. Joubert. "From ultrasoft pseudopotentials to the projector augmented-wave method". In: Phys. Rev. B 59.3 (1999), p. 1758.

[74] A. V. Ruban and I. A. Abrikosov. "Configurational thermodynamics of alloys from first principles: effective cluster interactions". In: Reports Prog. Phys. 71.4 (Apr. 2008), p. 046501. 
[75] A. Zunger et al. "Special quasirandom structures". In: Phys. Rev. Lett. 65.3 (1990), pp. 353-356.

[76] J. M. Sanchez, F. Ducastelle, and D. Gratias. "Generalized cluster description of multicomponent systems". In: Phys. A Stat. Mech. its Appl. 128.1 (1984), pp. 334-350.

[77] J. M. Sanchez. "Cluster expansions and the configurational energy of alloys". In: Phys. Rev. B 48.18 (1993), pp. 13-15.

[78] F. Tasnádi, M. Odén, and I. A. Abrikosov. "Ab initio elastic tensor of cubic $\mathrm{Ti}_{0.5} \mathrm{Al}_{0.5} \mathrm{~N}$ alloys: Dependence of elastic constants on size and shape of the supercell model and their convergence". In: Phys. Rev. B 85.14 (Apr. 2012), p. 144112.

[79] D. A. Porter and K. E. Easterling. Phase Transformations in Metals and Alloys, Third Edition (Revised Reprint). 1992.

[80] M. Finnis. Interatomic Forces in Condensed Matter. Oxford Series on Materials Modelling. OUP Oxford, 2003.

[81] A. E. Romanov et al. "Strain-induced polarization in wurtzite III-nitride semipolar layers". In: J. Appl. Phys. 100.2 (July 2006), p. 023522.

[82] T. Furukawa. "Piezoelectricity and pyroelectricity in polymers". In: Electr. Insul. IEEE Trans. 24.3 (1989), pp. 375-394.

[83] F. Bernardini, V. Fiorentini, and D. Vanderbilt. "Spontaneous polarization and piezoelectric constants of III-V nitrides". In: Phys. Rev. B 56.condmat/9705105. 16 (1997), p. 10024.

[84] R. Resta. "Macroscopic polarization in crystalline dielectrics: the geometric phase approach". In: Rev. Mod. Phys. 66.3 (1994).

[85] D. Vanderbilt. "Berry-phase theory of proper piezoelectric response". In: $J$. Phys. Chem. Solids 61.2 (Feb. 2000), pp. 147-151.

[86] D. Xiao, M.-C. Chang, and Q. Niu. "Berry phase effects on electronic properties". In: Rev. Mod. Phys. 82.3 (July 2010), pp. 1959-2007.

[87] W. Jiang et al. "Characterization of piezoelectric materials with large piezoelectric and electromechanical coupling coefficients." In: Ultrasonics 41.2 (Mar. 2003), pp. 55-63.

[88] A. Fick. "Ueber Diffusion". In: Ann. der Phys. und Chemie 170.1 (1855), pp. 59-86.

[89] A. Fick. "On liquid diffusion". In: Philos. Mag. Ser. 410.63 (1855), pp. 3039.

[90] P. Heitjans and J. Kärger. Diffusion in Condensed Matter. Ed. by Paul Heitjans and Jörg Kärger. 1st ed. Berlin/Heidelberg: Springer-Verlag, 2005.

[91] G. Mills, H. Jónsson, and G. K. Schenter. "Reversible work transition state theory: application to dissociative adsorption of hydrogen". In: Surf. Sci. 324.2-3 (1995), pp. 305-337. 
[92] G. Henkelman, B. P. Uberuaga, and H. Jónsson. "A climbing image nudged elastic band method for finding saddle points and minimum energy paths". In: J. Chem. Phys. 113.22 (2000).

[93] D. Sheppard, R. Terrell, and G. Henkelman. "Nudged elastic band method for finding minimum energy paths of transitions". In: J. Chem. Phys. 128.13 (Apr. 2008), p. 134106.

[94] J. D. Wrigley, M. E. Twigg, and G. Ehrlich. "Lattice walks by long jumps". In: J. Chem. Phys. 93.4 (Aug. 1990), p. 2885.

[95] A. Kolmogoroff. "Über die analytischen Methoden in der Wahrscheinlichkeitsrechnung". In: Math. Ann. 104.1 (1931), pp. 415-458.

[96] C. Tholander. "Ab initio piezoelectricity and energy landscape of $\mathrm{Y}_{0.375} \mathrm{Al}_{0.626} \mathrm{~N}$ ". MA thesis. Linköping University, 2011. 
CHAPTER 3

\section{Results and included pappers}

The results obtained from my research leading up to this thesis are presented in the form of four scientific papers published in international peer-reviewed journals. 


\subsection{List of publications}

[I] $\mathbf{Y}_{x} \mathbf{A l}_{1-x} \mathbf{N}$ thin films

A. Zukauskaite, C. Tholander, J. Palisaitis, P.O.A. Persson, V. Darakchieva, N. Ben Sedrine, F. Tasnádi, B. Alling, J. Birch, and L. Hultman

Journal of Physics D: Applied Physics, 45, 422001 (2012).

[II] Volume matching condition to establish the enhanced piezoelectricity in ternary $(\mathbf{S c}, \mathbf{Y})_{0.5}(\mathrm{Al}, \mathrm{Ga}, \mathbf{I n})_{0.5} \mathbf{N}$ alloys

C. Tholander, I.A. Abrikosov, L. Hultman, and F. Tasnádi

Physical Review B, 87, 94107 (2013).

[III] Effect of $\mathrm{Al}$ substitution on $\mathrm{Ti}, \mathrm{Al}$, and $\mathrm{N}$ adatom dynamics on TiN(0 01 ), (0 11 ), and (1 111$)$ surfaces

C. Tholander, B. Alling, F. Tasnádi, J.E. Greene, and L. Hultman

Surface Science, 630, 28 (2014).

[IV] Configurational disorder effects on adatom mobilities on $\mathbf{T i}_{1-x} A \mathbf{l}_{x} \mathbf{N}(001)$ surfaces from first principles

B. Alling, P. Steneteg, C. Tholander, F. Tasnádi, I. Petrov, J.E. Greene, and L. Hultman

Physical Review B, 85, 245422 (2012). 


\subsection{Summary of included papers}

\section{Paper I}

\section{$\mathbf{Y}_{x} \mathbf{A l}_{1-x} \mathbf{N}$ thin films}

\section{Summary}

An experimental and theoretical investigation of $\mathrm{Y}_{x} \mathrm{Al}_{1-x} \mathrm{~N}$ thin films. The theoretical work include $\mathrm{Y}_{x} \mathrm{Al}_{1-x} \mathrm{~N}$ lattice parameters, and phase stability calculations for cubic, hexagonal, and wurtzite phases in the range $0 \leq x \leq 1$. We show that it is possible to synthesize the wurtzite structure, both with theory and experiment.

\section{Author's contribution}

I performed all of the theoretical work, took part in discussion of the results, and wrote the parts in the manuscript relating to the theoretical calculations.

\section{Paper II}

Volume matching condition to establish the enhanced piezoelectricity in ternary $(\mathrm{Sc}, \mathrm{Y})_{0.5}(\mathrm{Al}, \mathrm{Ga}, \mathrm{In})_{0.5} \mathrm{~N}$ alloys

\section{Summary}

This work is a continuation of the work presented in my Master's thesis ${ }^{1}$. Here, we performed a search for new candidates for high temperature piezoelectric devices, guided by the giant piezoelectric increase found in ScAlN. I conclude that volume matching the parent materials is key to obtaining a giant piezoelectric increase in these material systems.

\section{Author's contribution}

In this work, I took part in planning, performed all the calculations, participated in the evaluation and interpretation of the results, and was responsible for writing the manuscript.

\footnotetext{
${ }^{1}$ Ref. [96]
} 


\section{Paper III}

Effect of Al substitution on Ti, Al, and $\mathbf{N}$ adatom dynamics on $\mathbf{T i N}\left(\begin{array}{lll}0 & 0\end{array}\right)$, (011), and (111) surfaces

\section{Summary}

In this article, I used DFT to investigate the effect of $\mathrm{Al}$ substitution on $\mathrm{Ti}, \mathrm{Al}$, and $\mathrm{N}$ adatom dynamics on $\mathrm{TiN}\left(\begin{array}{lll}0 & 0 & 1\end{array}\right),\left(\begin{array}{lll}0 & 1 & 1\end{array}\right)$, and (1 111$)$ surfaces. It is found that in general adatom mobilities are fastest on $\operatorname{TiN}(001)$, slower on $\operatorname{TiN}(111)$, and

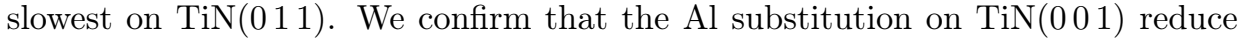
the $\mathrm{Ti}$ migration rate with little reduction to the $\mathrm{Al}$ migration. The effect was also found to be the opposite on $\mathrm{TiN}(111)$. In addition, we show that magnetic effects have a significant impact on the Ti adatom binding energies and diffusion path.

\section{Author's contribution}

I took part in planning of this project, performed the calculations, participated in the evaluation and interpretation of the results, and was responsible for writing the manuscript.

\section{Paper IV}

Configurational disorder effects on adatom mobilities on $\mathrm{Ti}_{1-x} \mathrm{Al}_{x} \mathrm{~N}(001)$ surfaces from first principles

\section{Summary}

This paper, is a study on the configurational disorder effects on adatom mobilities on $\mathrm{Ti}_{1-x} \mathrm{Al}_{x} \mathrm{~N}(001)$ surface by comparing the potential energy landscapes of $\mathrm{Ti}$ and $\mathrm{Al}$ adsorption on these surfaces. We conclude that the disorder reduce the $\mathrm{Ti}$ mobility, while $\mathrm{Al}$ mobilities experience only small reductions.

\section{Author's contribution}

For this work, I performed convergence tests of the structures used, and also took part in the discussion of the results. 


\section{Included Papers}

The articles associated with this thesis have been removed for copyright reasons. For more details about these see:

http://urn.kb.se/resolve?urn=urn:nbn:se:liu:diva- 110363 